\title{
Global tectonic patterns of the Moon
}

\author{
I. Matsuyama $^{\text {a, }}$, J.T. Keane ${ }^{b}$, A. Trinh ${ }^{a}$, M. Beuthe ${ }^{c}$, T.R. Watters ${ }^{d}$ \\ ${ }^{a}$ Lunar and Planetary Laboratory, University of Arizona, Tucson, AZ, USA \\ ${ }^{\mathrm{b}}$ California Institute of Technology, Pasadena, CA, USA \\ ${ }^{\mathrm{c}}$ Royal Observatory of Belgium, Brussels 1180, Belgium \\ d Center for Earth and Planetary Studies, Smithsonian Institution, Washington, DC, USA
}

\section{A R T I C L E I N F O}

\section{Keywords:}

Moon tectonics tides

Solid body rotational dynamics fossil figure

\begin{abstract}
A B S T R A C T
Planetary tectonics provide a record of the myriad of processes that shape planetary surfaces and interiors. While there is a long history of mapping and modeling planetary tectonics, stresses from disparate processes are not generally captured by a single model. We present a comprehensive and general stress and tectonics model that can consider multiple stress-generating mechanisms simultaneously. The model is applicable to mass loading with arbitrary geometry, rotational and orbital perturbations, and arbitrary elastic lithosphere thicknesses. This wholistic approach to tectonic modeling has important implications for understanding both lunar evolution and tectonics across the solar system. We apply this model to the Moon, which exhibits a global pattern of thrust faults. The ubiquitous presence of young thrust faults suggests that isotropic contraction plays a dominant role. However, their non-random orientation requires additional stress-generating mechanisms that are not isotropic. Best-fit solutions correspond to models combining isotropic contraction with orbit recession, despinning, and South Pole-Aitken ejecta loading and the corresponding true polar wander. Contraction and despinning assuming an elastic shell with a thinner equatorial region can lead to misfits that are smaller than those assuming a constant thickness elastic shell. The young age of the faults favors recent contraction and recession; however, unrelaxed stresses from older processes combined with recent contractional stresses can also generate young faults. This possibility is supported by the Moon's ability to preserve a fossil figure.
\end{abstract}

\section{Introduction}

A myriad of processes can shape planetary surfaces and interiors, generating observable tectonic patterns. Previous studies have provided methods for computing stresses associated with rotational perturbations (Melosh 1977, 1980; Matsuyama and Nimmo 2008), tidal perturbations (Melosh 1977; Cheng and Toksoz 1978; Melosh 1980; Harada and Kurita 2006; Matsuyama and Nimmo 2008; Wahr et al. 2009), and surface mass loading perturbations (Turcotte et al. 1981; Banerdt et al. 1982; Willemann and Turcotte 1982; Janes and Melosh 1990). Stresses from disparate processes can affect tectonic patterns; however, none of these methods are general enough to consider multiple processes simultaneously. We develop a comprehensive and general stress and tectonics model that can consider multiple stress-generating mechanisms simultaneously. Additionally, our method is not limited by assumptions of elastic lithosphere thickness and incompressibility. The stresses and corresponding tectonic patterns can be computed for any process given its gravitational potential.
We apply the model to the Moon, which exhibits a global scale pattern of thrust faults indicative of a global stress scenario where horizontal principal stresses are compressive. Lobate thrust fault scarps were first detected on the Moon in high-resolution Apollo Panoramic Camera and some Lunar Orbiter photographs (Schultz 1976; Binder 1982). Because of their small scale, the full extent of the distribution and number of fault scarps was not realized until the Lunar Reconnaissance Orbiter (LRO) mission (Watters et al. 2010). Ongoing surveys using LRO Camera images have revealed a globally distributed population of over 3500 thrust fault scarps (Watters et al. 2015a, 2019). Lunar scarps are an order of magnitude smaller in scale than the large-scale lobate scarps on Mercury and Mars, averaging tens of meters of relief with lengths of only up to tens of kilometers (Banks et al. 2012). The scale, pristine appearance, relations with small craters, and preserved small superposed graben, indicate the fault scarps are very young, active within the last 100 Myr and possibly still active (Watters et al., 2010, 2015, 2019; Clark et al. 2017; van der Bogert et al. 2018). Fig. 1a shows the observed global pattern by sampling the digitized segments in 400 equal area

\footnotetext{
* Corresponding author.

E-mail address: isa@lpl.arizona.edu (I. Matsuyama).
} 
regions partitioned using the "igloo" method of Leopardi (2006). Polar faults tend to be more $E$-W oriented, while equatorial faults tend to be more N-S oriented (Watters et al. 2015a; Fig. 1b). Although the effect of illumination bias cannot be completely discounted, the large range of orientations at all latitudes suggests that the pattern of orientations is not controlled by Sun azimuth (Watters et al. 2015a).

The contractional strain estimated from the observed thrust faults suggests a minimum radius decrease $\sim 25 \mathrm{~m}$ (Watters et al. 2015a), which corresponds to a minimum isotropic stress $\sim 2 \mathrm{MPa}$ (Eq. (15)) assuming an unbroken lithosphere and ignoring stress relaxation as faults form. As the Moon cools its volume decreases, generating compressional horizontal stresses. Thermal evolution models starting with an initially molten Moon predict a radius decrease $\sim 16 \mathrm{~km}$ over the past 4 Gyr (Solomon and Chaiken 1976; Solomon 1986). Models starting with a $\sim 200-300 \mathrm{~km}$ thick near-surface magma ocean predict a smaller radius decrease $\lesssim 1 \mathrm{~km}$ over the past $\sim 2 \mathrm{Gyr}$ due to the tradeoff between expansion of the inner regions and contraction of the outer regions (Solomon and Chaiken 1976; Solomon 1977).

The ubiquitous presence of thrust faults indicates that contraction plays a dominant role. Assuming isotropic contraction alone, the magnitudes of the horizontal compressive principal stresses are equal, producing randomly oriented thrust faults. Therefore, the observed nonrandom orientations require non-isotropic processes that can generate additional global-scale stresses. Several such processes can influence the observed pattern of thrust faults: orbit recession, despinning, tides raised by the Earth on the Moon, surface mass loading, and true polar wander (TPW, the reorientation of the rotation and tidal axes relative to the surface (Melosh 1975; Keane and Matsuyama 2014)). A notable feature of the Moon that can affect the global tectonic pattern is its large rotational deformation (which creates a rotational bulge) and tidal deformation (which creates a tidal bulge along the tidal axis, the axis connecting the centers of the Earth and the Moon). The long-wavelength lunar figure is triaxial, as expected due to these deformations; however, the observed deformation is significantly larger than the expected deformation assuming a hydrostatic response to the present rotational and tidal potentials. This discrepancy indicates the presence of a fossil figure, frozen in when the Moon was closer to Earth and the rotational and tidal potentials were larger (Jeffreys 1915; Lambeck and Pullan 1980; Garrick-Bethell et al. 2006; Matsuyama 2013; Keane and Matsuyama 2014; Qin et al. 2018). As the Moon recedes from the Earth, it deforms in response to changes in the rotational and tidal potentials, and the existence of a fossil figure requires the preservation of stresses associated with past, larger rotational and tidal deformation.

The model presented in this paper captures all the processes discussed above, and we evaluate which of these processes considered individually or jointly, most likely generated the observed tectonic pattern. The rest of the paper is organized as follows: In $\S 2$ we review the theoretical background and describe the model and theoretical method. $\S 3$ goes through the most important loading stresses on the Moon - SPA ejecta (§3.1) and mare flood basalts ( $\$ 3.2)$ - and the tectonic patterns that result when combining those loading stresses with orbital, rotational, and fossil figure stresses ( $\$ 3.3$ ). We compare the results to the observed lunar tectonic pattern in $\S 3.3$, and present conclusions in $\S 4$.

\section{Theory}

\subsection{Gravitational potential}

The gravitational potential $\Phi$ at a surface point with spherical coordinates $(R, \theta, \phi)$, where $R$ is the mean radius, $\theta$ is colatitude and $\phi$ is longitude, can be expanded in surface spherical harmonics with unnormalized expansion coefficients $\Phi_{\ell m}$ as (e.g., Wieczorek 2015)

$\Phi(r, \theta, \phi)=\frac{G M}{R}+\frac{G M}{R} \sum_{\ell=0}^{\infty} \sum_{m=-\ell}^{\ell} \Phi_{\ell m} Y_{\ell m}(\theta, \phi)$,

where $G$ is the gravitational constant and $M$ is the body's mass. The spherical harmonic functions $Y_{\ell m}$ of degree $\ell$ and order $m$ are defined as

$Y_{\ell m}(\theta, \phi)=\left(\begin{array}{cc}P_{\ell m}(\cos \theta) \cos (m \phi) & \text { if } m \geq 0 \\ P_{\ell|m|}(\cos \theta) \sin (|m| \phi), & \text { if } m<0\end{array}\right.$

where $P_{\ell m}$ is the unnormalized associated Legendre function (e.g., Arfken and Weber 1995; Wieczorek 2015). We adopt the sign convention of geodesy and astronomy in which the gravitational potential is positive. On the right-hand-side (RHS) in Eq. (1), the first term is the first-order expression for a spherically symmetric state, and the second term corresponds to perturbations from this state. Below, we consider the specific case of rotational, orbital, and surface loading perturbations.

\subsubsection{Rotational and tidal perturbations}

Rotational and tidal perturbations to the gravitational potential due to a change from an initial state with rotation rate $\Omega_{i}$, rotation axis with spherical coordinates $\left(\theta_{R, i} \phi_{R, i}\right)$, semimajor axis $a_{i}$, and tidal axis with spherical coordinates $\left(\theta_{T, i}, \phi_{T, i}\right)$, to a final state with rotation rate $\Omega_{f}$, rotation axis with spherical coordinates $\left(\theta_{R, f}, \phi_{R, f}\right)$, semimajor axis $a_{f}$, and tidal axis with spherical coordinates $\left(\theta_{T, f}, \phi_{T, f}\right)$ can be written as (Matsuyama et al. 2014)

$\Phi_{2 m} \equiv \Phi_{2 m}^{f}-\Phi_{2 m}^{i}$

where a

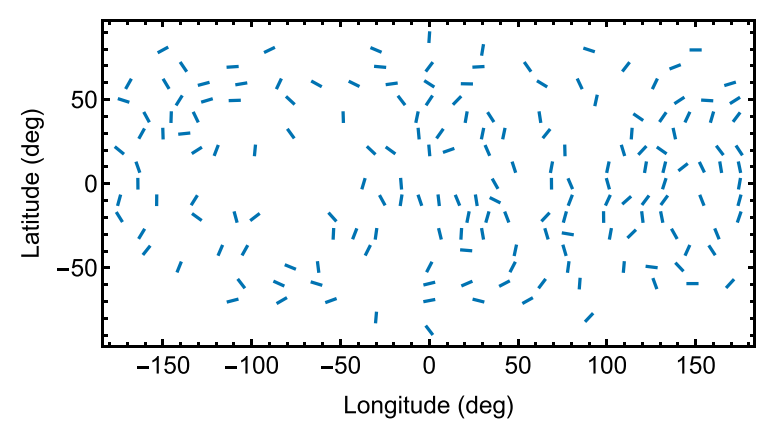

b

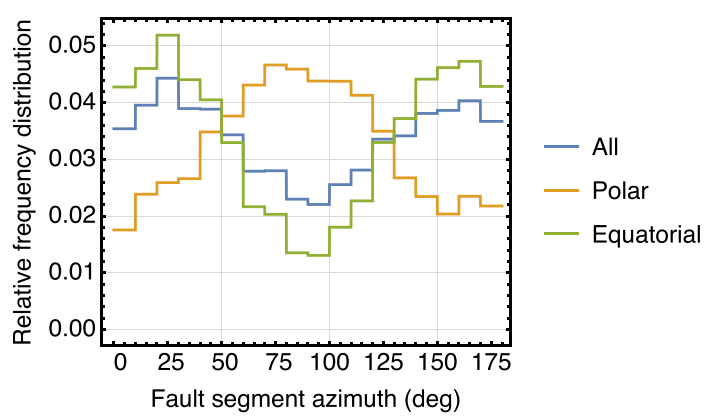

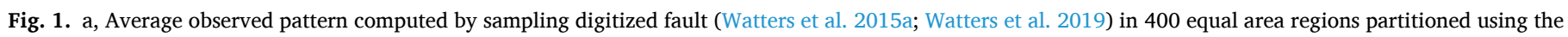

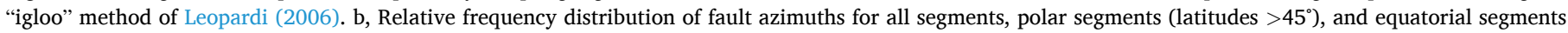

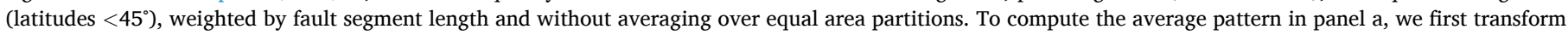

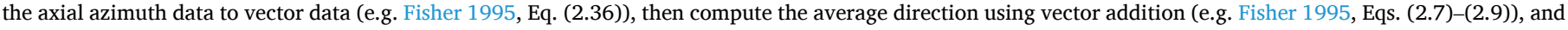
finally back-transform the vector data to axial azimuth data by dividing by a factor of two. 
$\Phi_{2 m}^{s}=\left(2-\delta_{m 0}\right) \frac{(2-|m|) !}{(2+|m|) !}\left[\Phi_{20}^{R^{\prime}} Y_{2 m}\left(\theta_{R, s}, \phi_{R, s}\right)+\Phi_{20}^{T^{\prime}} Y_{2 m}\left(\theta_{T, s}, \phi_{T, s}\right)\right]$,

$\delta_{m 0}$ is the Kronecker delta function, and we use a superscript or subscript to indicate the initial $(s=i)$ and final $(s=f)$ states. Assuming synchronous rotation and small orbital eccentricity at the initial and final states $\left(e_{i} \ll 1\right.$ and $\left.e_{f} \ll 1\right)$,

$\Phi_{20}^{R^{\prime}}=-\frac{\Omega_{s}^{2} R^{3}}{3 G M}$

$\Phi_{20}^{T^{\prime}}=\frac{M_{T}}{M}\left(\frac{R}{a_{s}}\right)^{3}$,

where $M_{T}$ is the mass of the tide-raising body. Note that we account for the possibility of changes in the rotation rate between the initial and final states $\left(\Omega_{i} \neq \Omega_{f}\right)$. More generally, assuming a rotation rate to mean motion $\left(n_{s}\right)$ ratio $p_{s} \equiv \Omega_{s} / n_{s}$, the orbit-averaged potential coefficients are

$\Phi_{20}^{R^{\prime}}=-\frac{\Omega_{s}^{2} R^{3}}{3 G M}+\frac{1}{2} \frac{M_{T}}{M}\left(\frac{R}{a_{s}}\right)^{3}\left[H\left(p_{s}, e_{s}\right)-\left(1-e_{s}^{2}\right)^{-3 / 2}\right]$

$\Phi_{20}^{T^{\prime}}=\frac{M_{T}}{M}\left(\frac{R}{a_{s}}\right)^{3} H\left(p_{s}, e_{s}\right)$,

where $H\left(p_{s}, e_{s}\right)$ are Hansen coefficients, which describe the timeaveraged gravitational potential as a function of spin-orbit resonance ratio $p_{s}$ and orbital eccentricity $e_{s}$ (Goldreich 1966; Matsuyama and Nimmo 2009). Once again, we use a subscript to indicate the initial ( $s=$ $i)$ and final $(s=f)$ states. We consider the orbit-averaged potential coefficients because we are interested in rotational and tidal perturbations over time scales that are much longer than the orbital period. Assuming synchronous rotation and small eccentricity, $p_{s}=1, H(1, e)=1-5 e^{2} / 2$ $+\cdots \sim 1$, and we can recover Eq. (5) as expected. Assuming a 3:2 spinorbit resonance (Mercury's present spin-orbit state) and small eccentricity, $p_{s}=3 / 2, H(3 / 2, e)=7 e / 2+\cdots \ll 1$, and the axially symmetric deformation around the tidal axis becomes negligible compared with the axially symmetric deformation around the rotation axis $\left(\Phi_{20}^{T \prime} \ll \Phi_{20}^{R^{\prime}}\right)$. The difference between these two cases is more easily understood when considering the motion of the Earth as seen in a reference frame centered on the Moon and rotating with the Moon's mean rotation rate, $\Omega_{s}=p_{s} n_{s}$. In synchronous rotation, the Earth remains near the tidal axis at all times (Murray and Dermott 1999, Fig. 5.1), producing an orbit-averaged tidal bulge along the tidal axis. In 3:2 spin-orbit resonance, the Earth revolves around the Moon (Murray and Dermott 1999, Fig. 5.10), producing a small orbit-averaged tidal bulge.

Eqs. (5) and (6) can be used to compute the gravitational potential perturbations if the initial and final rotational and orbital parameters are known. Given the large, non-hydrostatic gravity field of the Moon suggesting the presence of a fossil figure (Jeffreys 1915; Lambeck and Pullan 1980; Garrick-Bethell et al. 2006; Matsuyama 2013; Keane and Matsuyama 2014; Qin et al. 2018), it is useful to consider the gravitational potential perturbations associated with it. We extend the approach of Matsuyama and Nimmo (2009) and Matsuyama et al. (2014) to describe any fossil figure contribution as the combination of two axially symmetric perturbations around two orthogonal axes, as expected for the combination of rotational and tidal deformation. Given the degree-2 potential coefficients of the fossil figure when it is aligned with the present rotation and tidal axes, $\Phi_{20}^{F F}$ and $\Phi_{22}^{F F}$, the forcing gravitational potential perturbation responsible for producing the fossil figure can be written as $\Phi_{2 m}=\Phi_{2 m}^{s}$, where the potential coefficients $\Phi_{2 m}^{s}$ are given by Eq. (4) with

$$
\begin{aligned}
& \Phi_{20}^{R^{\prime}}=\frac{\Phi_{20}^{F F}+2 \Phi_{22}^{F F}}{k_{2}^{T^{*}}-k_{2}^{T}} \\
& \Phi_{20}^{T^{\prime}}=\frac{4 \Phi_{22}^{F F}}{k_{2}^{T^{*}}-k_{2}^{T^{*}}} .
\end{aligned}
$$

In this equation, $\Phi_{20}^{R^{\prime}}$ and $\Phi_{20}^{T^{\prime}}$ quantify the axially symmetric deformation around the rotation and tidal axes, and $k_{2}^{T}$ and $k_{2}^{T *}$ are degree-2 tidal Love numbers with and without an elastic lithosphere respectively (Matsuyama et al. 2014). These tidal Love numbers describe the deformation in response to tidal forcing and are given by the ratio between the response and forcing gravitational potentials. For example, $k_{2}^{T}=0$ in the limiting case of an infinite rigidity elastic lithosphere and no deformation. We compute the tidal Love numbers using the propagator matrix method (Appendix C).

Given $\Phi_{20}^{R^{\prime}}$ and $\Phi_{20}^{T}$, Eq. (4) can be used to compute the degree-2 gravity coefficients for any fossil figure orientation, and Eq. (7) allows us to compute $\Phi_{20}^{R^{\prime}}$ and $\Phi_{20}^{T}$ for arbitrary axisymmetric perturbations given $\Phi_{20}^{F F}$ and $\Phi_{22}^{F F}$. For a non-resonant spin-orbit state, the long-term deformation becomes axisymmetric around the rotation axis and $\Phi_{22}^{F F}$ $=\Phi_{20}^{T}=0$. The potential coefficients of the fossil figure are given by $\left(k_{2}^{T *}\right.$ $\left.-k_{2}^{T}\right) \Phi_{2 m}$. For example, for a fossil figure aligned with the present rotation and tidal axes (i.e. $\theta_{R, s}=\phi_{T, s}=0^{\circ}$ and $\left.\theta_{T, s}=90^{\circ}\right),\left(k_{2}^{T_{*}^{*}}-\right.$ $\left.k_{2}^{T}\right) \Phi_{20}=\Phi_{20}^{F F}$ and $\left(k_{2}^{T * *}-k_{2}^{T}\right) \Phi_{22}=\Phi_{22}^{F F}$, as expected.

\subsubsection{Surface loading perturbations}

Mass loading perturbations due to a surface load with density $\rho_{L}$ and thickness

$H(\theta, \phi)=R \sum_{\ell=1}^{\infty} \sum_{m=-\ell}^{\ell} H_{\ell m} Y_{\ell m}(\theta, \phi)$

can be written as (Matsuyama et al. 2014)

$\Phi_{\ell m}=\left(1+k_{\ell}^{L}\right)\left(\frac{3}{2 \ell+1}\right) \frac{\rho_{L}}{\bar{\rho}} H_{\ell m}$

where $k_{\ell}^{L}$ is the degree- $\ell$ load Love number, $\bar{\rho}$ is the mean density of the planet, and $H_{\ell m}$ is a dimensionless expansion coefficient. Similar to tidal Love numbers, load Love numbers describe the deformation in response to mass loading and are given by the ratio between the response and forcing gravitational potentials. For example, if the load is fully compensated $k_{\ell}^{L}=-1$, such that the total (forcing plus response) gravitational potential is zero, and $k_{\ell}^{L}=0$ in the limiting case of an infinite rigidity elastic lithosphere and no deformation. We compute the load Love numbers using the propagator matrix method (Appendix C).

Planetary surface loading perturbations are often nearly axially symmetric, especially at low harmonic degrees for which perturbations are largest. Therefore, it is useful to consider this special case. If we define

$H_{\ell 0}^{\prime} \equiv \frac{2 \ell+1}{4 \pi R} \int_{0}^{2 \pi} \int_{0}^{\pi} d \theta d \phi \sin \theta H(\theta) P_{\ell 0}(\cos \theta)$

as the expansion coefficient for the case when the load is centered on the north pole, the expansion coefficients for the same load centered at $\left(\theta_{L}, \phi_{L}\right)$ are given by (Matsuyama et al. 2014)

$H_{\ell m}=H_{\ell 0}^{\prime}\left(2-\delta_{m 0}\right) \frac{(\ell-|m|) !}{(\ell+|m|) !} Y_{\ell m}\left(\theta_{L,}, \phi_{L}\right)$

\subsection{Stresses}

Given the potential coefficients of the perturbations due to rotational, tidal, fossil figure, or surface loading perturbations, the corresponding displacements at the planet surface $(r=R)$ are

$\left(d_{r}, d_{\theta}, d_{\phi}\right)=R \sum_{\ell=0}^{\infty} \sum_{m=-\ell}^{\ell}\left(h_{\ell}, l_{\ell} \partial_{\theta}, \frac{1}{\sin \theta} l_{\ell} \partial_{\phi}\right) \Phi_{\ell m} Y_{\ell m}$,

where $h_{\ell}$ and $l_{\ell}$ are tidal displacement Love numbers for rotational, tidal, or fossil figure perturbations; and loading displacement Love numbers for surface loading perturbations. These Love numbers 
generally depend on the perturbation forcing time scale. For example, for long-term perturbations, the relevant Love numbers are the longterm Love numbers; and for diurnal forcing perturbations, the relevant Love numbers are the Love numbers at the tidal forcing period. In this paper, we consider long-term perturbations that depend on long-term Love numbers. We compute the tidal and load displacement Love numbers using the propagator matrix method (Appendix C).

Assuming a compressible, elastic lithosphere with Lamé parameters $\mu$ and $\lambda$, the Lagrangian increment to the Cauchy stress tensor is given by (Appendix A)

$$
\begin{aligned}
\tau_{\theta \theta} & =\frac{\lambda}{\lambda+2 \mu} \tau_{r r}+\frac{2 \mu}{R(\lambda+2 \mu)}\left[(3 \lambda+2 \mu) d_{r}+2(\lambda+\mu) \partial_{\theta} d_{\theta}+\lambda\left(\frac{\partial_{\phi} d_{\phi}}{\sin \theta}+d_{\theta} \cot \theta\right)\right] \\
\tau_{\phi \phi} & =\frac{\lambda}{\lambda+2 \mu} \tau_{r r}+\frac{2 \mu}{R(\lambda+2 \mu)}\left[(3 \lambda+2 \mu) d_{r}+\lambda \partial_{\theta} d_{\theta}+2(\lambda+\mu)\left(\frac{\partial_{\phi} d_{\phi}}{\sin \theta}+d_{\theta} \cot \theta\right)\right] \\
\tau_{\theta \phi} & =\frac{\mu}{R}\left(\partial_{\theta} d_{\phi}+\frac{\partial_{\phi} d_{\theta}}{\sin \theta}-d_{\phi} \cot \theta\right)
\end{aligned}
$$

At the surface $(r=R), \tau_{r r}=0$ for rotational or tidal perturbations, and

$\tau_{r r}=-\rho_{L} g H=-\rho_{L} g R \sum_{\ell=1}^{\infty} \sum_{m=-\ell}^{\ell} H_{\ell m} Y_{\ell m}$,

where $g$ is the surface gravity, for surface loading. Eq. (13) is equivalent to Eqs. (B.8)-(B.10) in Wahr et al. (2009) for rotational or tidal stresses at the surface with $\tau_{r r}=0$. For an isotropic contraction, $\partial_{\theta}=\partial_{\phi}=d_{\theta}=d_{\phi}=$ 0 and

$\tau_{\theta \theta}=\tau_{\phi \phi}=\frac{\lambda}{\lambda+2 \mu} \tau_{r r}+\frac{2 \mu(3 \lambda+2 \mu)}{(\lambda+2 \mu)} \frac{d_{r}}{R}$,

where $\tau_{r r}=0$ at the planet surface $(r=R)$. This expression is equivalent to Eq. (25) of Melosh (1977) for contraction stresses at the planet surface.

Given the stress tensor, the expected tectonic patterns can be found using Anderson's theory of faulting (Anderson 1951). The model is general enough to be applicable to mass loading, and rotational and tidal perturbations. Additionally, it is not restricted by assumptions on the interior structure (including arbitrary elastic lithosphere thicknesses), load size, or load geometry, as long as the interior is spherically symmetric.

\subsection{Equatorial lithosphere thinning}

Mercury exhibits a non-random, global pattern of scarps similar to the one on the Moon (Watters et al. 2015b), and an elastic lithosphere with a thinner equatorial region due to insolation driven variations has been suggested as a possible explanation for the non-random orientation of the faults (Williams et al. 2011; Watters et al. 2015b). The Moon and Mercury experience a different magnitude but a similar latitudinal variation in surface temperature (e.g. Vasavada et al. 1999, Fig. 5); therefore, similar variations of elastic lithosphere thickness are expected (Williams et al. 2011). We consider this effect using the thin elastic shell model of Beuthe (2010) because the model described in $\S 2$ assumes a uniform elastic lithosphere.

A pattern with equatorial N-S oriented faults and polar $E$-W oriented faults can arise due to despinning and contraction on an elastic lithosphere that is thinner at the equator (Beuthe 2010, Fig. 8). Given the qualitative agreement between this pattern and observed lunar pattern (Fig. 4f), we focus on this case, which arises when the equatorial thin zone is more extended than the one for a model with a thickness perturbation $\propto P_{20}(\cos \theta)$ (Beuthe, 2010). This last assumption is supported by the results of Williams et al. (2011), who computed for Mercury a variation of the lithospheric thickness corresponding to this definition of an extended equatorial thin zone (Williams et al. 2011, Fig. 4). Other cases such as orbital recession or TPW can be treated with the non-uniform thin shell method (Beuthe 2018) by replacing the despinning potential by the static potential perturbation derived in section $\S 2.1 .1$.

We note that contraction of an elastic lithosphere thinner at the poles generates N-S oriented faults on the entire surface, the same pattern as the one due to despinning and contraction on a uniform lithosphere (Fig. 4e). This lithospheric thickness variation could be caused by tidal dissipation driven by eccentricity tides when the ancient lithosphere was underlaid by a magma ocean during the early evolution of the Moon (e. g. Garrick-Bethell et al. 2010).

\section{Application to the Moon}

We apply the model and theoretical methods described in $\S 2$ to the Moon assuming interior structure parameters based on mass, moment of inertia, gravity and topography constraints (Matsuyama et al. 2016). These parameters are summarized in Table 1 but it is worth noting that the predicted tectonic patterns are not sensitive to them.

The evolution of the lunar orbit and the corresponding rotational and tidal perturbations depend on mutual tides and energy dissipation in the Earth-Moon system (e.g. Goldreich 1966). The present orbit recession rate is constrained by lunar laser ranging to $3.82 \pm 0.07 \mathrm{~cm} \mathrm{yr}^{-1}$ (Dickey et al. 1994), and the mean orbit recession rate over the past $\sim 600 \mathrm{Myr}$ is constrained by Earth's paleorotation values inferred from geological observations to $2.17 \pm 0.31 \mathrm{~cm} \mathrm{yr}^{-1}$ (Williams 2000). Earlier orbit recession rate estimates based on geologic constraints are more uncertain, and theoretical estimates are limited due to uncertainties in tidal energy dissipation at this time. Nevertheless, it is possible to constrain the lunar orbit at earlier times if the present, anomalously large rotational and tidal deformation of the Moon is interpreted as due to a fossil figure preserving a record of past orbital parameters (Jeffreys 1915; Lambeck and Pullan 1980; Garrick-Bethell et al. 2006). For reasonable effective rigidities (which depend on the thickness and rigidity of the elastic lithosphere), the observed fossil figure requires initial semimajor axes $\lesssim 20 R_{\oplus}$ (the present semimajor axis is $60 R_{\oplus}$ ) (Matsuyama 2013; Qin et al. 2018) and initial orbital eccentricities $\sim 0.5$ (Matsuyama 2013). Such a high eccentricity would generate extreme tidal heating, complicating the preservation of the fossil figure (Meyer et al. 2010). Impact basins can contribute significantly to the fossil figure, and removing their contribution lowers the required initial orbital eccentricity to $\sim 0.2$ (Keane and Matsuyama 2014). Additionally, the removal of impact basin contributions reveals a fossil figure that is misaligned relative to the present principal axes of inertia (Keane and Matsuyama 2014), suggesting an early TPW driven by mass loading associated with impact basins (Melosh 1975). The most important loading stresses are associated with SPA ejecta and mare flood basalts, which we describe below.

\subsection{SPA ejecta}

SPA ejecta represents a substantial load on the lunar farside highlands, plausibly contributing several kilometers to the overall crustal

Table 1

Lunar interior structure parameters. We assume a $25 \mathrm{~km}$ thick elastic lithosphere. Since we are interested in the long-term Love numbers, the rigidity of all layers is set to zero except for the elastic lithosphere (see Appendix C). The Lamé parameter $\lambda$ of the elastic lithosphere is $40 \mathrm{GPa}$, which corresponds to a Poisson ratio of 0.25 .

\begin{tabular}{llllll}
\hline & $\begin{array}{l}\text { Solid } \\
\text { core }\end{array}$ & $\begin{array}{l}\text { Liquid } \\
\text { core }\end{array}$ & Mantle & Lithosphere & $\begin{array}{l}\text { Elastic } \\
\text { lithosphere }\end{array}$ \\
\hline $\begin{array}{c}\text { Radius }(\mathrm{km}) \\
\begin{array}{c}\text { Density }(\mathrm{kg} \\
\mathrm{m}^{-3} \text { ) }\end{array}\end{array}$ & 203 & 381 & 1697 & 1712 & 1737 \\
$\begin{array}{c}\text { Shear modulus, } \\
\mu(\mathrm{GPa})\end{array}$ & 0 & 0 & 0 & 0 & 2740 \\
\hline
\end{tabular}


thickness (Fig. 2a; Melosh et al. 2017). At present, the spatial distribution of this ejecta is poorly constrained - in part because other impact basins also contribute to the crustal structure of the farside highlands. In order to remove the effects of smaller impact basins, we applied the crustal reconstruction method of Bouley et al. (2020) to isolate the crustal structure of SPA. This approach utilizes crustal thickness maps derived from gravity and topography data (Wieczorek et al. 2013). In this technique, we assume that impact basins modify the crust in a way that is both symmetric about the basin center, and that approximately conserves crustal mass. Crustal volume is calculated by scaling and combining crustal thickness, porosity, and density-which are constrained from geophysical data and analysis of returned samples (Wieczorek et al. 2013). Fig. 2b shows an example of this fitting process for the Orientale impact basin. For each impact basin we calculate the azimuthally-averaged crustal thickness as a function of distance from the center of the basin. We then iteratively search for a mean crustal a

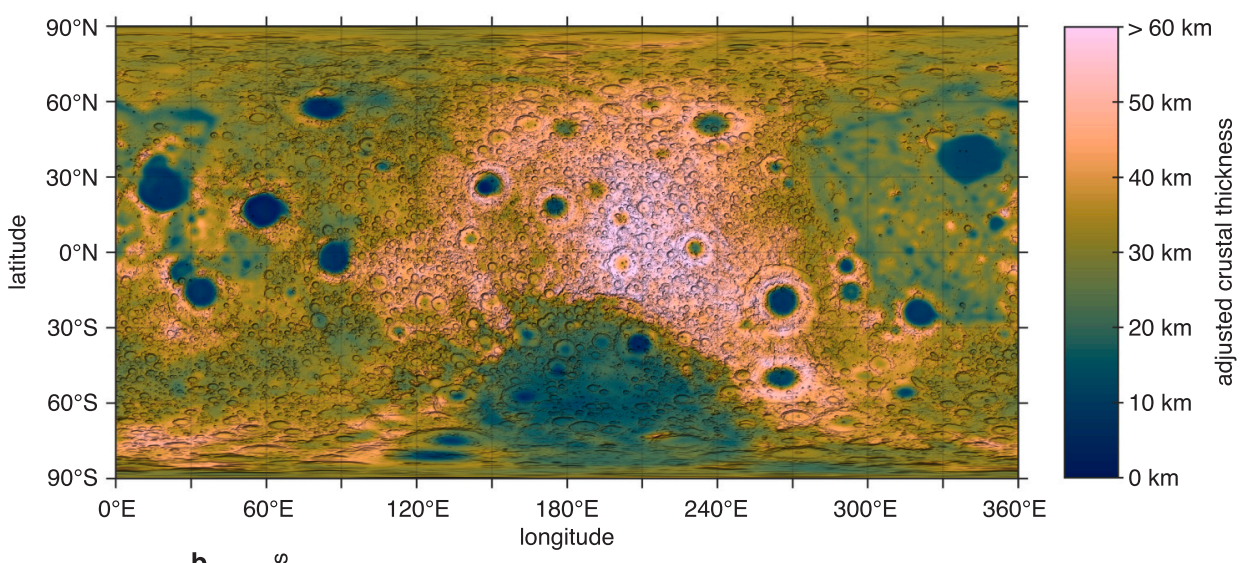

b

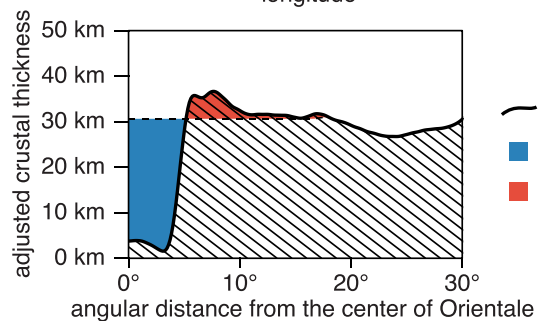

c

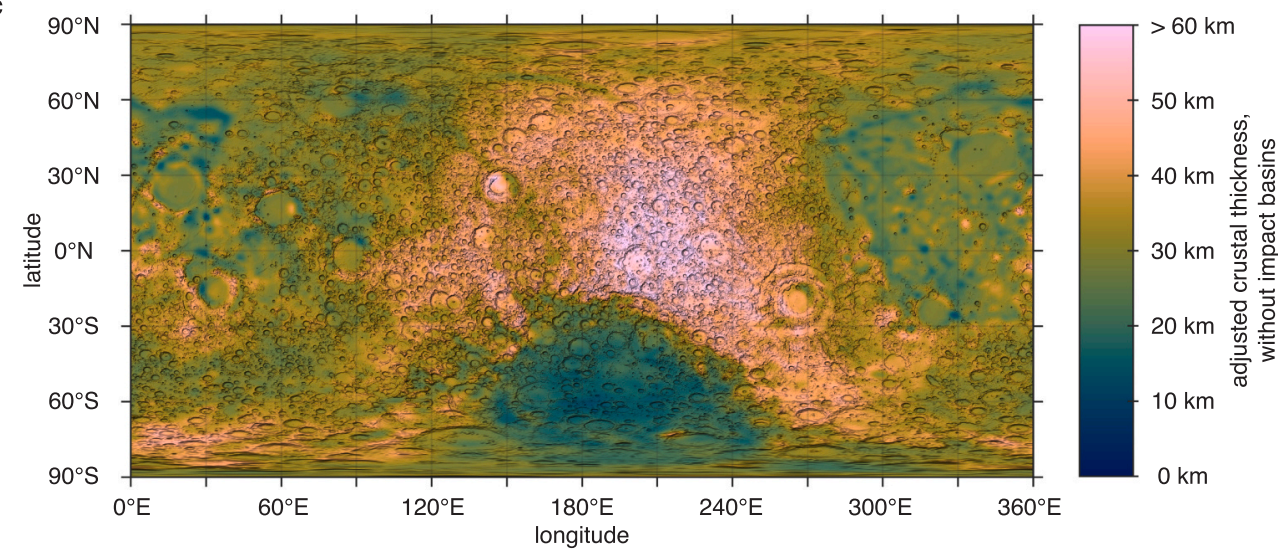

d

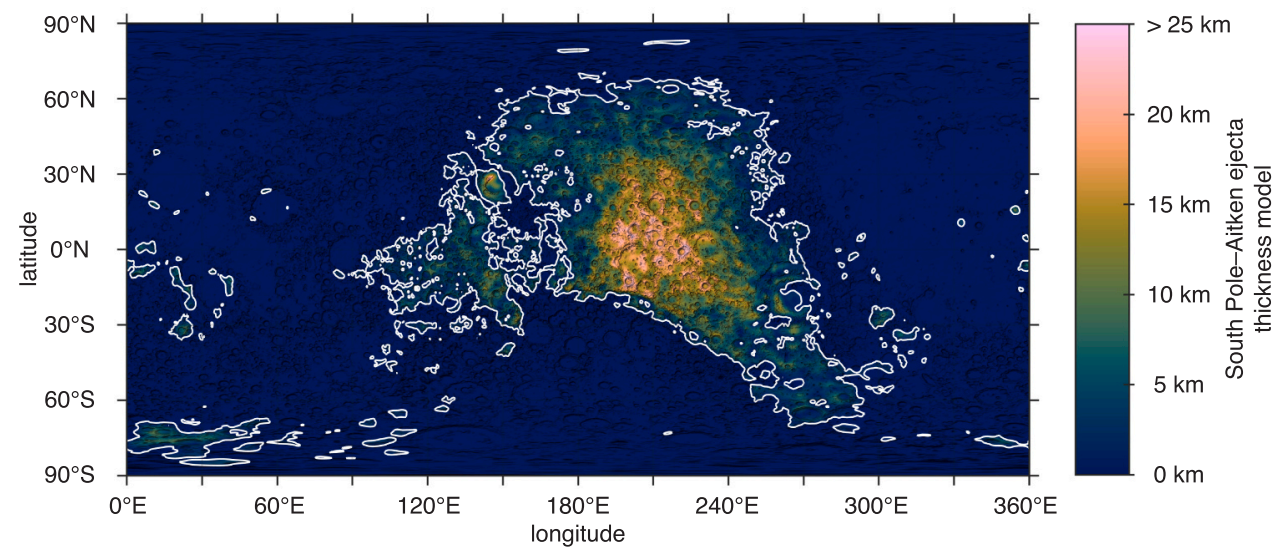

Fig. 2. a, Adjusted crustal thickness of the Moon (model 1 from Wieczorek et al. 2013). Adjusted crustal thickness is crustal thickness, but scaled by the crustal density and porosity so that the density is globally uniform. This is a small effect, but is useful as it enables reconstruction of crustal structure by conservation of mass arguments (Bouley et al. 2020). b, The azimuthally-averaged crustal structure about the Orientale impact basin (solid black line with hatching below). We calculate the pre-Orientale crustal thickness by assuming that the basin reshaped crustal structure in a way that conserved mass, i.e., the mass deficit (blue) is equal to the mass excess (red). The pre-Orientale crustal thickness is the dashed line. c, Crustal structure without the contribution of the 73 largest lunar impact basins. d, Our model for the spatial distribution and volume of SPA impact ejecta. This is merely the adjusted crustal thickness above $40 \mathrm{~km}$ in Fig. 2c. The volume excess in this region is equal to the volume deficit in SPA. (For interpretation of the references to color in this figure legend, the reader is referred to the web version of this article.) 
thickness that balanced the volume deficit in the central depression with the volume excess in the surrounding terrains associated with crustal thickening and ejecta deposits. More complete description of this method can be found in Bouley et al. (2020). We used this method to sequentially remove the 73 largest lunar impact basins identified by gravity and topography data (Neumann et al. 2015), with the exception of SPA and the putative Procellarum impact basin (which may not be an impact basin; Andrews-Hanna et al. 2014).

Fig. 2c shows the crustal structure of the Moon with the 73 largest impact basins (other than SPA) removed. Removing impact basins reveals a more subdued crustal thickness map, dominated by the crustal thinning in SPA, a crustal excess in the farside highlands, and a polygonal region of thin crust in the Procellarum region- likely associated with the unique tectono-magmatic evolution of that region (AndrewsHanna et al. 2014). The excess crustal thickness in the farside highlands is interesting for two reasons. First, the spatial distribution of this crustal excess is similar to the expected spatial distribution of SPA ejecta (Melosh et al. 2017), where the ejecta is deposited in a non-trivial pattern downrange of the impact (which likely impacted from south to north; e.g., Wieczorek et al. 2012). Second, the volume excess in this region is approximately equal to the volume deficit in the SPA depression (a fact that has been recognized since the first global topography maps of the Moon; Zuber et al. 1994). This evidence supports the hypothesis that a significant component of the farside highlands is made up of SPA ejecta. We define a SPA ejecta blanket model from this "corrected" crustal thickness map by assuming that all crust thicker than 40 $\mathrm{km}$ arises from SPA ejecta (Fig. 2d). The $40 \mathrm{~km}$ thickness arises from assuming that the volume excess in this ejecta blanket is balanced by the volume deficit in SPA.

The inferred SPA crustal thickness model (Fig. 2d) is equivalent to an ejecta thickness model if loading is the only mechanism involved. However, given the long-wavelength nature of the model, fossil figure (Keane and Matsuyama 2014) and tidal dissipation (Garrick-Bethell et al. 2014) perturbations may contribute significantly. For all practical purposes, fossil figure perturbations are limited to degree two, and tidal dissipation perturbations are limited to degrees two and four (Beuthe 2013). Therefore, we can find a best-fit SPA ejecta thickness model using the long-wavelength gravity field, excluding degrees two and four, as a constraint. For simplicity, we assume that the SPA ejecta thickness scales linearly with the crustal thickness derived above and obtain a best-fit solution by minimizing the misfit

$\chi_{S P A}^{2}=\sum_{m=-3}^{3}\left(\frac{\Phi_{\ell m}^{O B S}-\Phi_{\ell m}^{S P A}}{\delta \Phi_{\ell m}^{O B S}}\right)^{2}+\sum_{\ell=5}^{\ell_{\max }} \sum_{m=-\ell}^{\ell}\left(\frac{\Phi_{\ell m}^{O B S}-\Phi_{\ell m}^{S P A}}{\delta \Phi_{\ell m}^{O B S}}\right)^{2}$.

Here, SPA potential coefficients are given by Eq. (9) with $H_{\ell m}=$ $\alpha h_{\ell m}$, where $\alpha$ is a scaling constant, $h_{\ell m}$ are the crustal thickness expansion coefficients, $\Phi_{\ell m}^{O B S}$ are the observed potential coefficients, and $\delta \Phi_{\ell m}^{O B S}$ are the corresponding uncertainties. SPA ejecta compensation is taken into account by the term $1+k_{\ell}^{L}$ in $\Phi_{\ell m}^{S P A}$ (Eq. (9)), where $k_{\ell}^{L}$ is the degree- $\ell$ load Love number. We use the JPL Lunar gravity field GRAIL420C1A (Konopliv et al. 2013) for the observed potential coefficients. The misfit minimization yields $0.55 \leq \alpha \leq 0.58$ for $7 \leq \ell_{\max } \leq$ 15 and we adopt $\alpha=0.55$. In principle, it is possible to model possible fossil figure and tidal dissipation contributions to constrain the SPA ejecta thickness. However, this is beyond the scope of this paper.

The mass redistribution associated with SPA ejecta is capable of driving TPW (Melosh 1975; Keane and Matsuyama 2014). The nonspherical part of the inertia tensor which controls TPW is directly related to the degree-2 potential coefficients (e.g. Eq. (3) of Matsuyama et al. 2014). Therefore, it is possible to compute the TPW associated with any perturbation given its degree- 2 potential coefficients. The SPA ejecta potential coefficients are given by Eq. (9) with the best-fit solution discussed above. The total, non-spherical part of the inertia tensor can be written as $I_{i j}=I_{i j}^{S P A}+I_{i j}^{F F}+I_{i j}^{E Q}$, where $I_{i j}^{S P A}$ is the SPA ejecta contribution, $I_{i j}^{F F}$ is a fossil figure contribution, and $I_{i j}^{E Q}$ is an equilibrium contribution associated with the response to the present rotational and tidal potentials (Matsuyama et al. 2014). The fossil figure contribution can be found given the total inertia tensor $I_{i j}$ (which can be found using the observed degree- 2 potential coefficients), the SPA ejecta contribution, and the equilibrium contribution (e.g. Matsuyama et al. 2014, Eqs. (19) and (27))). The rotation and tidal axis locations prior to TPW can then be calculated by diagonalizing $I_{i j}^{F F}$, and the coefficients for the gravitational potential perturbation responsible for the fossil figure can be found using Eqs. (4) and (7). This yields an initial rotation axis at $68^{\circ}$ $\mathrm{N}, 215^{\circ} \mathrm{E}$ and an initial tidal axis at $6^{\circ} \mathrm{N}, 320^{\circ} \mathrm{E}$ recorded by a fossil figure, which is similar to the TPW solution inferred by Keane and Matsuyama (2014) with an initial rotation axis at $72^{\circ} \mathrm{N}, 222^{\circ} \mathrm{E}$ and an initial tidal axis at $14^{\circ} \mathrm{N}, 2^{\circ} \mathrm{E}$. The initial rotation axis locations are the same within a few degrees, and the difference between the initial tidal axis locations is larger. These differences are not surprising because Keane and Matsuyama (2014) assumed an axially symmetric SPA ejecta while we use the observed crustal thickness to construct a non-axially symmetric SPA ejecta model.

\subsection{Mare}

While ejecta from SPA dominates the loading of the lunar farside, lunar maria dominate the nearside. Maria are the extensive, flood basalts that superpose low-lying terrains and impact basins- predominantly on the lunar nearside, and within the Procellarum KREEP terrain (PKT, where KREEP stands for potassium, rare-earth elements, and phosphorus; Jolliff et al. 2000). The areal extent of the mare has been mapped with great detail by the LRO Wide Angle Camera (Nelson et al. 2014). The total thickness and volume of the mare have been inferred from a number of geological and geophysical methods, and modern estimates generally point to thicknesses of $\sim 1 \mathrm{~km}$ (Gong et al. 2016), although there is considerable uncertainty.

We use the LRO-derived map of mare boundaries, expand it in spherical harmonics, and find the best-fit mare load thickness by minimizing the misfit to the observed gravity field, after correcting for the SPA contribution. That is, we obtain a best-fit solution by minimizing the misfit

$$
\chi_{\text {Mare }}^{2}=\sum_{m=-3}^{3}\left(\frac{\Phi_{\ell m}^{O B S}-\Phi_{\ell m}^{S P A}-\Phi_{\ell m}^{M A R}}{\delta \Phi_{\ell m}^{O B S}}\right)^{2}+\sum_{\ell=5}^{\ell_{\max }} \sum_{m=-\ell}^{\ell}\left(\frac{\Phi_{\ell m}^{O B S}-\Phi_{\ell m}^{S P A}-\Phi_{\ell m}^{M A R}}{\delta \Phi_{\ell m}^{O B S}}\right)^{2},
$$

where we adopt the SPA solution described above for $\Phi_{\ell m}^{S P A}$ and the maria potential coefficients $\Phi_{\ell m}^{M A R}$ are given by Eq. (9) with the maria load thickness coefficients $H_{\ell m}^{M A R}$. These coefficients are

$H_{\ell m}^{M A R} \equiv\left(2-\delta_{m 0}\right) \frac{2 \ell+1}{4 \pi R} \frac{(\ell-|m|) !}{(\ell+|m|) !} \int_{0}^{2 \pi} \int_{0}^{\pi} d \theta d \phi \sin \theta H^{M A R}(\theta, \phi) Y_{\ell m}(\theta, \phi)$,

where we assume a constant value for $H^{\mathrm{MAR}}(\theta, \phi)$ at the locations highlighted in red in Fig. 3 and $H^{M A R}(\theta, \phi)=0$ elsewhere. As discussed above for the SPA ejecta thickness, we exclude degrees two and four in Eq. (17) to avoid fossil figure and tidal dissipation contributions to the observed gravity field. Minimization of Eq. (17) yields a best-fit constant thickness for the lunar maria. This best-fit solution is sensitive to the degree truncation, with maria thicknesses in the range $0.62-0.84 \mathrm{~km}$ for $20 \leq \ell_{\max } \leq 30$. We consider harmonic degrees higher than those considered for the SPA ejecta model given the smaller size of the maria. We arbitrarily choose a maria thickness of $0.78 \mathrm{~km}$ corresponding to the $\ell_{\max }=25$ solution; however, the tectonic patterns shown below are indistinguishable from each other for $20 \leq \ell_{\max } \leq 30$. It is worth noting that the compensated maria height is not uniform because longer wavelength contributions are more compensated, which is quantified by the load Love numbers in Eq. (9). Following the same procedure described above for the SPA ejecta, we find a small amount of TPW 


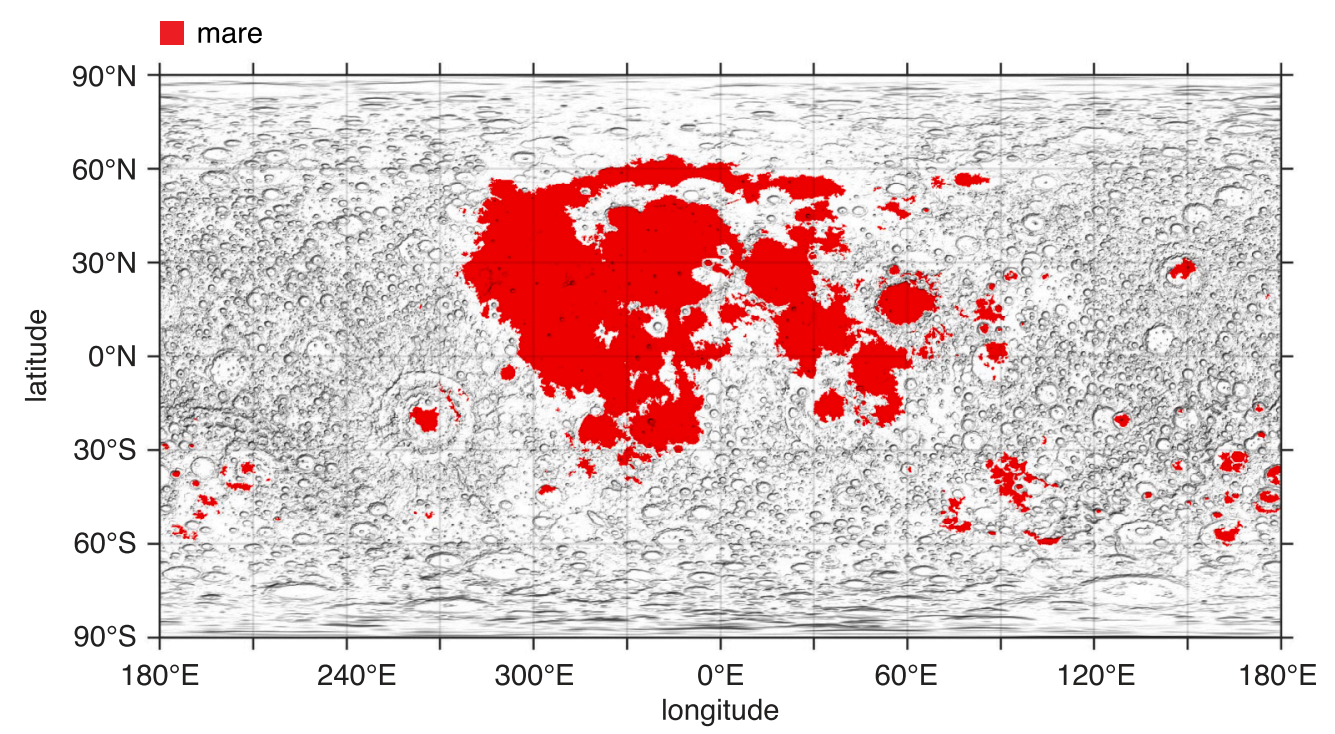

Fig. 3. Lunar Reconnaissance Orbiter-derived map of lunar maria (from Nelson et al. 2014).

driven by maria loading alone (ignoring SPA ejecta), with an initial rotation axis at $88.1^{\circ} \mathrm{N}, 327.1^{\circ} \mathrm{E}$ and an initial tidal axis at $1.5^{\circ} \mathrm{S}, 356.9^{\circ}$ E.

\subsection{Tectonic patterns}

Assuming isotropic contraction alone, the magnitudes of the horizontal compressive principal stresses are equal, producing randomly oriented thrust faults. The non-isotropic orbital, rotational, tidal, fossil figure, or surface loading perturbations generate horizontal principal stresses that can be compressive or extensional. In the cases considered below, we choose a global isotropic contraction that is large enough to make horizontal principal stresses compressional on the entire surface and produce widespread thrust faulting. In this case, contraction is responsible for the thrust style of faulting and the non-isotropic processes are responsible for the orientation of the faults.

Fig. 4 compares the observed tectonic pattern with the predicted patterns due to the combination of global contraction with rotational and orbital perturbations. The orbital and rotational evolution of the Moon is not well constrained due to its complicated dependence on poorly constrained energy dissipation processes in the Earth and the Moon (e.g. Goldreich 1966; Peale and Cassen 1978; Meyer et al. 2010; Qin et al. 2018). Therefore, we consider a wide range of orbital and rotational perturbations.

The symmetry of the predicted tectonic patterns can be explained by the symmetry of the forcing potential, i.e. the difference between the final and initial gravitational potentials averaged over the orbital period. For orbit recession with synchronous rotation throughout (Fig. 4a), the forcing potential is axially symmetric around the tidal axis, producing a deformation and a corresponding tectonic pattern with the same symmetry. For orbit recession with an initial 3:2 spin-orbit resonance (Fig. 4b), or despinning from a non-resonant spin-orbit state to synchronous rotation (Fig. 4d), the forcing potential is axially symmetric around an axis passing through the equator at $\pm 90^{\circ}$ longitudes, explaining the symmetry of the tectonic patterns.

Despinning from a non-resonant spin-orbit state to another nonresonant one produces N-S oriented faults on the entire surface (Fig. 4e), and polar faults become $E$-W oriented for an elastic shell with a thinner equatorial region (Fig. 4f). The forcing potential is axially symmetric around the rotation axis in this case, producing a deformation and corresponding tectonic pattern with the same symmetry if the shell is uniform. This case assumes that the tectonic pattern is established before the Moon arrives at a spin-orbit resonance state, the timing of which is poorly constrained.

TPW driven by internal mass anomalies can occur without leaving an observable surface feature. Therefore, we explore all possible TPW geometries and find a best-fit TPW solution by minimizing the misfit between the observed and predicted fault azimuths (Fig. 4c). This best-fit TPW solution corresponds to a reorientation of the tidal axis alone, with an initial tidal axis at the equator and $61^{\circ} \mathrm{E}$. The tectonic pattern is quasiconcentric around the initial tidal axis.

Fig. 5 compares the observed tectonic pattern with the predicted patterns due to the combination of global contraction with SPA ejecta loading, SPA ejecta and maria loading, and the corresponding TPW. We also consider the tectonic patterns due to loading or TPW alone (middle and bottom rows). These tectonic patterns are calculated using the models described in $\S 3.1$ and $\S 3.2$. SPA ejecta loading alone produces a tectonic pattern that is quasi-concentric around an axis passing through the center of SPA, roughly at the equator and $150^{\circ} \mathrm{W}$ (Fig. 5c). As discussed above, TPW driven by SPA ejecta loading reorients both the rotation and tidal axes, with an initial rotation axis at $68^{\circ} \mathrm{N}, 145^{\circ} \mathrm{W}$ and an initial tidal axis at $6^{\circ} \mathrm{N}, 40^{\circ} \mathrm{W}$. The TPW tectonic pattern is quasiconcentric around the initial tidal axis (Fig. 5e). Loading and TPW contributions are important when considering both mechanisms (Fig. 5a). Proximal to SPA, the pattern is dominated by SPA ejecta loading, with quasi-concentric faults around SPA. Distal to SPA, the pattern is dominated by TPW, with quasi-concentric faults around the initial tidal axis. Mare loading stresses are significantly smaller than SPA ejecta loading stresses, and their effect is only marginally noticeable when considering mass loading alone (e.g. compare Figs. $5 \mathrm{c}$ and $\mathrm{d}$ at $\sim 20^{\circ} \mathrm{N}, \sim 0^{\circ} \mathrm{E}$ ).

Figs. 4 and 5 provide a qualitative comparison between the observed and predicted tectonic patterns. We quantify the agreement between the observed and predicted patterns in Fig. 6 by computing the azimuth misfit $\delta$, defined as the absolute difference between the observed and predicted fault azimuths, and the global average misfit $\bar{\delta}$, defined as the global average of $\delta$ weighed by fault segment length. In these calculations, we use all $N=51614$ fault segments instead of the 400 average azimuths shown in Figs. 4 and 5 for the tectonic patterns. Assuming isotropic contraction alone, the expected distribution of the azimuth misfit is uniform (gray flat lines in Fig. 6), which corresponds to a mean misfit with a normal distribution with a mean value of $45^{\circ}$ and standard 
a

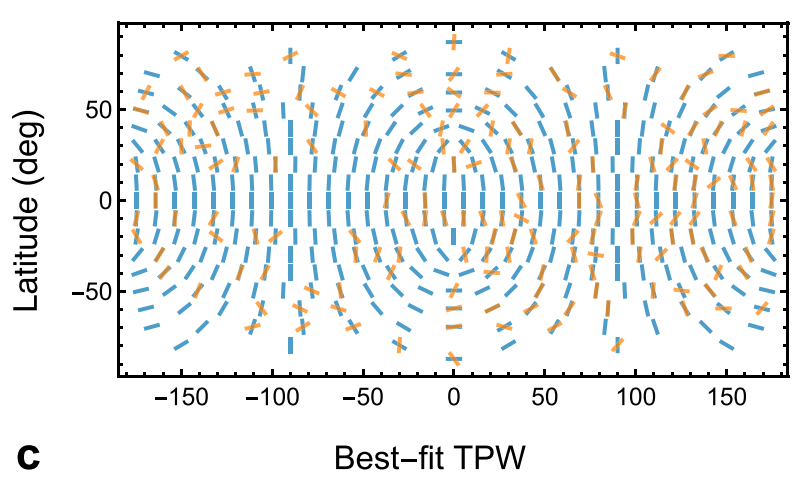

b

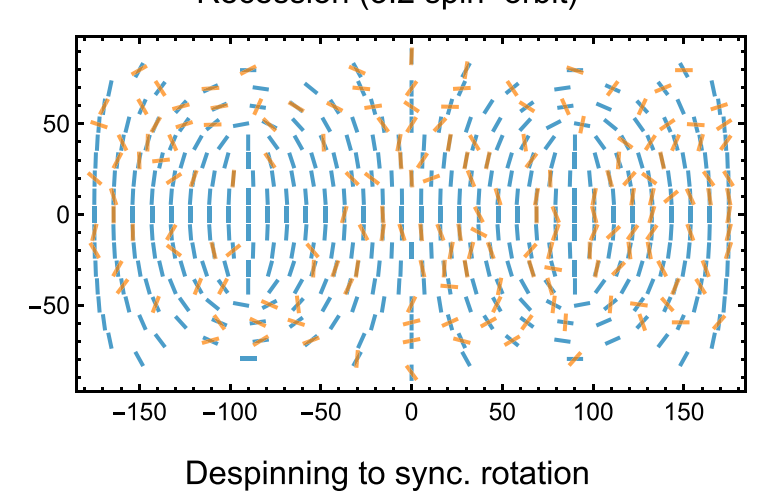

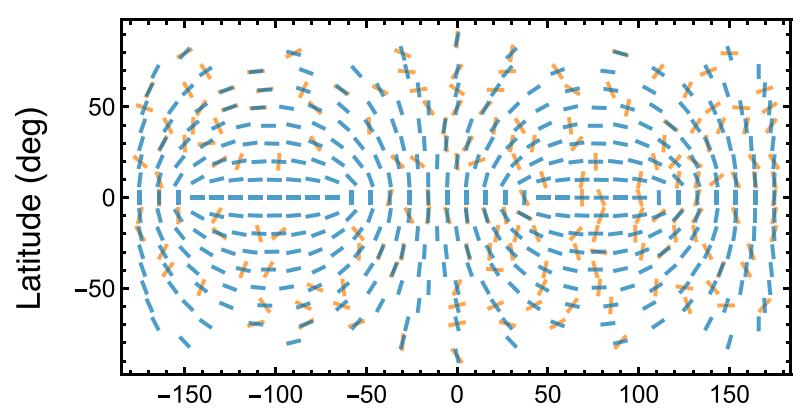

e Despinning, no resonance

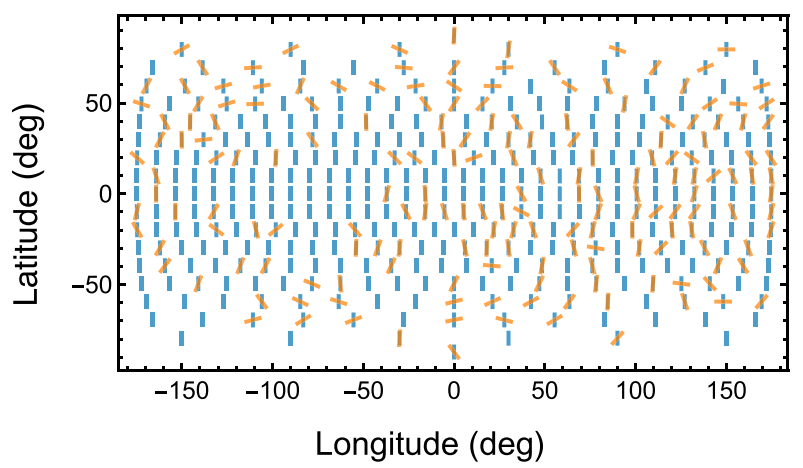

f Despinning, no resonance, non-uniform shell

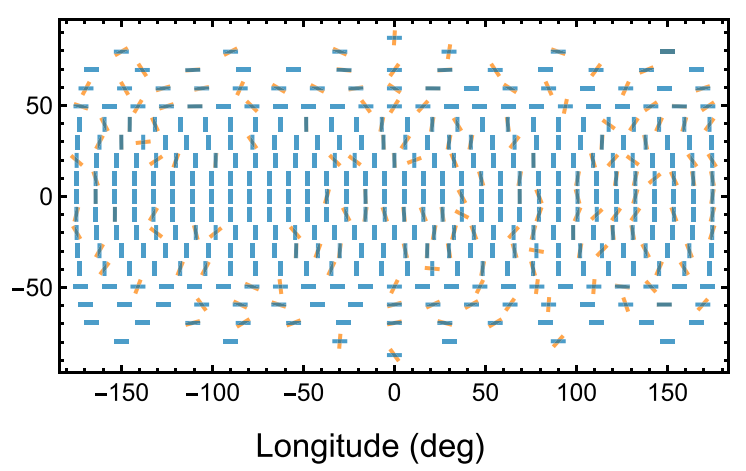

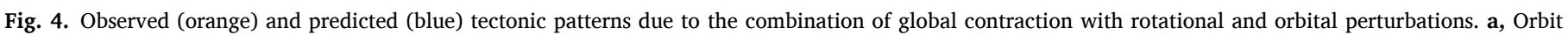

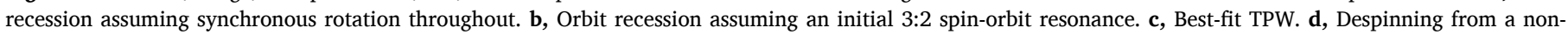

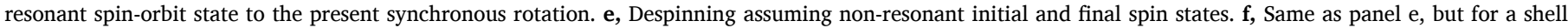

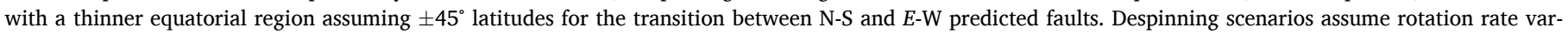

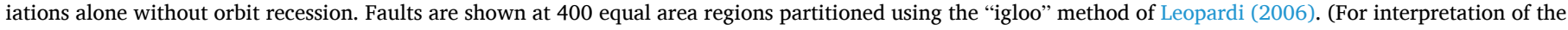
references to colour in this figure legend, the reader is referred to the web version of this article.)

deviation of $\sigma=90^{\circ} /(12 N)^{1 / 2}=0.1^{\circ}$. In this case, the probability of a mean misfit outside the range $45.0^{\circ} \pm 0.5^{\circ}$ is less than $0.002 \%$. As a caveat, we note that we are implicitly assuming that fault azimuths are random at any scale to compute the expected standard deviation. However, neighboring faults could start to affect their mutual azimuths at small scales. As a conservative estimate, we can use the 400 average azimuths (Figs. 4 and 5) to compute the expected standard deviation instead of using all fault segment azimuths, which corresponds to assuming that faults can be randomly oriented if they are separated by $\gtrsim 10^{\circ}$. In this case, the expected standard deviation is $\sigma=90^{\circ} /(12 \times$ $400)^{1 / 2}=1.3^{\circ}$.

Best-fit solutions correspond to orbit recession (Fig. 6a), despinning (Fig. 6b), SPA ejecta loading and the corresponding TPW (Fig. 6f), and despinning for an elastic shell with a thinner equatorial region (Fig. 6e). The mean misfit for these cases is smaller than $40^{\circ}$, which is well outside the expected value assuming isotropic contraction alone.

Orbit recession misfits are not sensitive to the assumed initial spinorbit state. Despite differences in the predicted tectonic patterns (4a), assuming different initial spin-orbit states produces similar misfits, with mean misfits $38.8^{\circ} \leq \bar{\delta} \leq 39.6$ (Fig. 6a). Despinning misfits are sensitive to the assumed initial and final spin-orbit state. Assuming non-resonant initial and final spin-orbit states results in a smaller misfit relative to the case assuming a non-resonant initial spin-orbit state and a final synchronous rotation state (Fig. $6 \mathrm{~b}, \bar{\delta}=39.5^{\circ}$ vs. $43.4^{\circ}$ ). SPA ejecta loading provides a smaller misfit than maria loading (Fig. $6 \mathrm{~d}, \bar{\delta}=40.8^{\circ}$ vs. $43.6^{\circ}$ ), and adding the expected SPA-driven TPW reduces the misfit (Fig. 6 f, $\bar{\delta}=39.3^{\circ}$ ).

The misfits for the TPW inferred by Keane and Matsuyama (2014) due to the mass redistribution associated with the largest impact basins, 
SPA

a

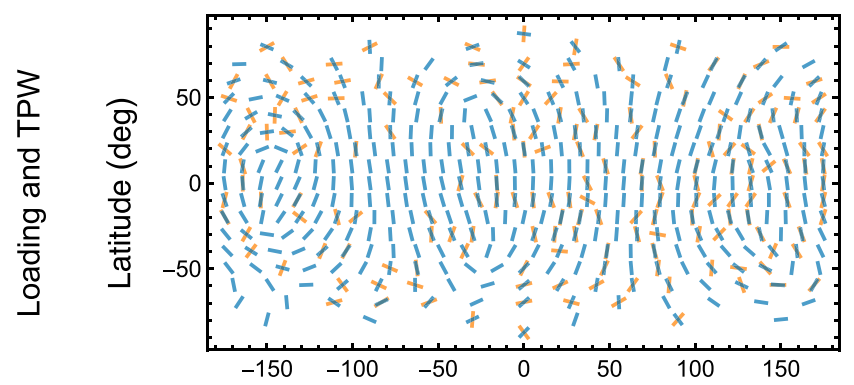

C

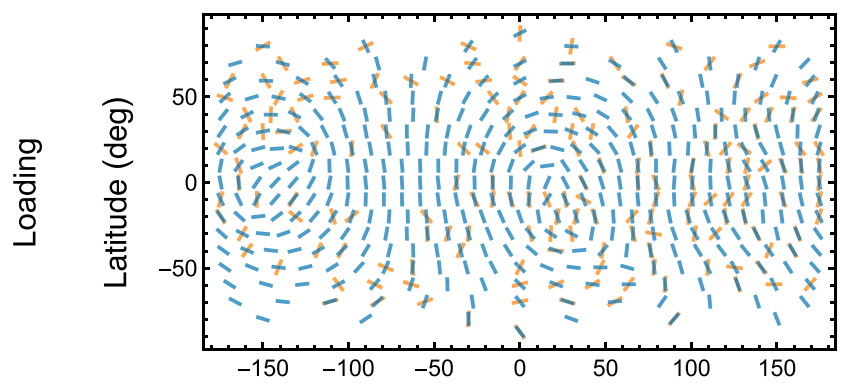

e

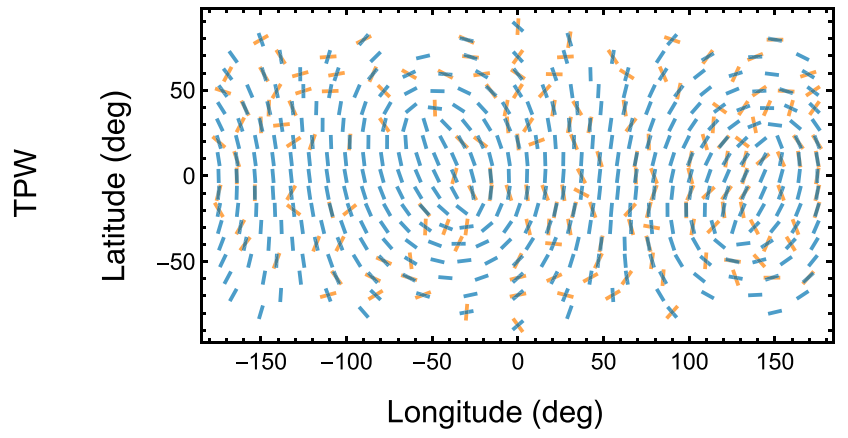

SPA and Mare

b

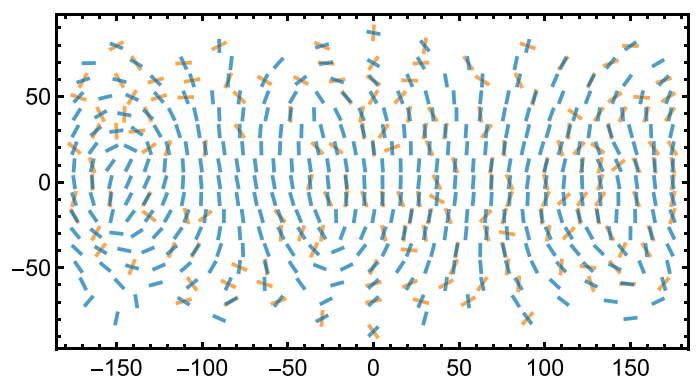

d

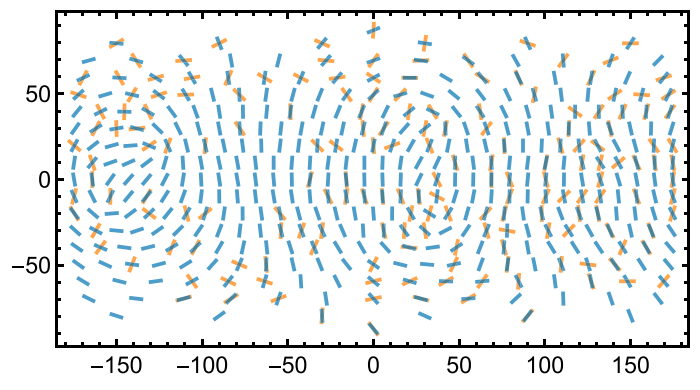

$\mathbf{f}$

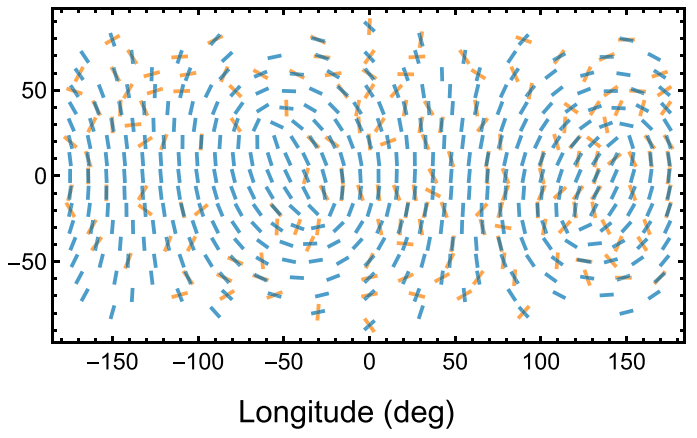

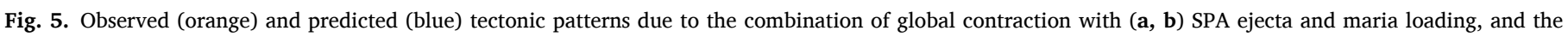

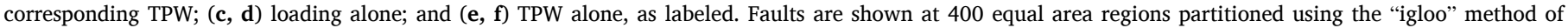
Leopardi (2006). (For interpretation of the references to colour in this figure legend, the reader is referred to the web version of this article.)

and the TPW inferred by Siegler et al. (2016) based on the distribution of antipodal hydrogen deposits are larger than or equal to (within the expected standard deviation) the isotropic contraction misfit (Fig. 6c, $\bar{\delta} \geq 45^{\circ}$ ). The best-fit TPW solution discussed above corresponding to a reorientation of the tidal axis alone (Fig. 4c) provides a smaller misfit (Fig. $6 \mathrm{c}, \bar{\delta}=43.5^{\circ}$ ). These TPW scenarios must be driven by mass redistribution, which can generate additional stresses that must be taken into account. As discussed above, SPA ejecta loading and the corresponding TPW, which has a similar TPW solution as the one found by Keane and Matsuyama (2014), leads to a smaller misfit (Fig. 6f, $\bar{\delta}=$ $\left.39.3^{\circ}\right)$.

As shown in Fig. 4f, the combination of contraction and despinning assuming non-resonant initial and final spin states for an elastic shell with an extended thinner equatorial region produces N-S oriented equatorial faults and E-W oriented polar faults. The transition latitude between N-S and E-W faults depends on the extent of the thinner equatorial region and the ratio between contraction and despinning (Beuthe 2010, Fig. 9). The former can be inferred from the insolation pattern (Williams et al. 2011); however, the latter is not constrained.
Therefore, we consider a range of transition latitudes. For transition latitudes $\sim 45^{\circ}-60^{\circ}$, the mean misfit becomes smaller than the misfit for the best-fit solutions discussed above assuming an elastic shell with constant thickness (Fig. 6 f, $\bar{\delta} \sim 36^{\circ}$ vs. $\sim 39^{\circ}$ ).

In addition to the fault orientation pattern, we consider the spatial distribution of faults and the magnitude of deviatoric stresses. Fig. 7 compares the spatial distribution of the observed fault segment length per unit area and the deviatoric stress. Faulting is more likely in regions with larger deviatoric stress; therefore, variations in the deviatoric stress can produce variations in the spatial distribution of faults. However, this correlation is not obvious for any of the best-fit models considered above. Watters et al. (2015a, 2019) note that diurnal stresses result in maximum compression around the sub-Earth and anti-Earth points, and that some shallow moonquakes in proximity to young fault scarps occur near when peak compressional stresses are reached at their locations. However, deviatoric stresses due to diurnal tides are maximum at the sub- and anti-Earth points only for a small fraction of the surface (Appendix E, Fig. 9). Additionally, their magnitude is $\sim 1 \mathrm{kPa}$ (Appendix E), which is orders of magnitude smaller than the magnitude of deviatoric 


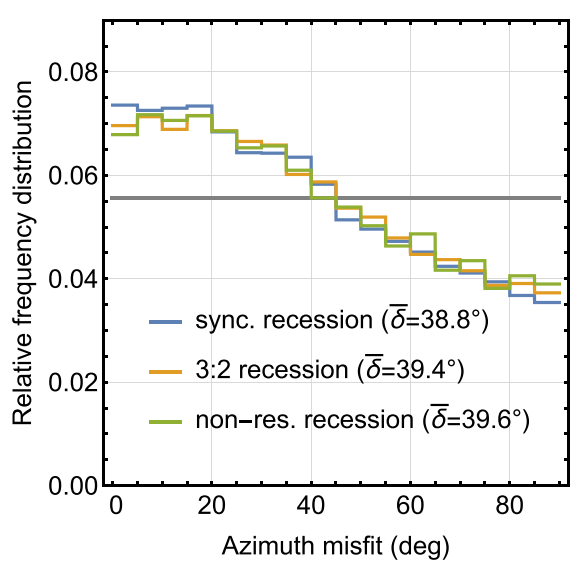

d

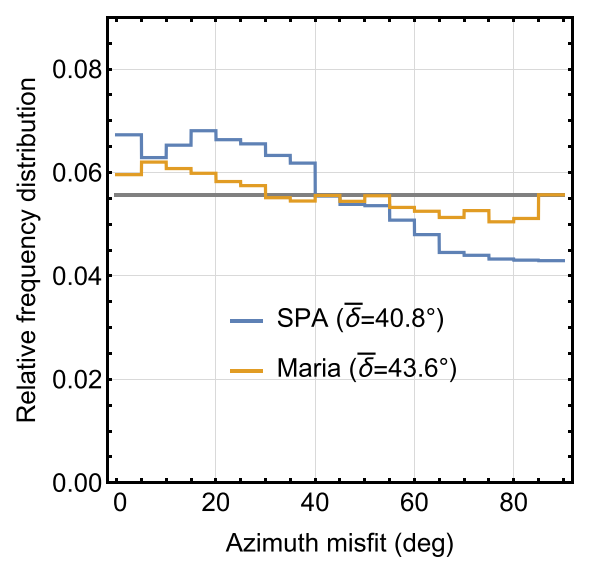

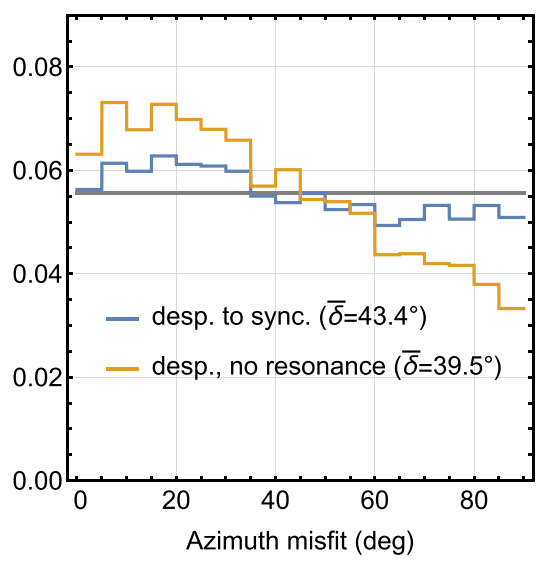

Equatorial shell thinning

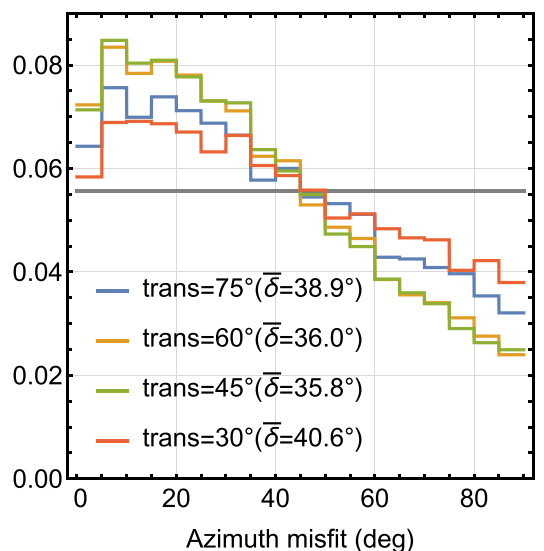

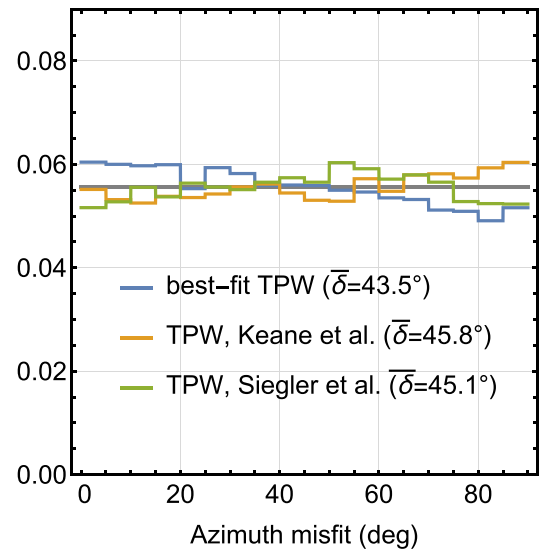

Best-fit solutions

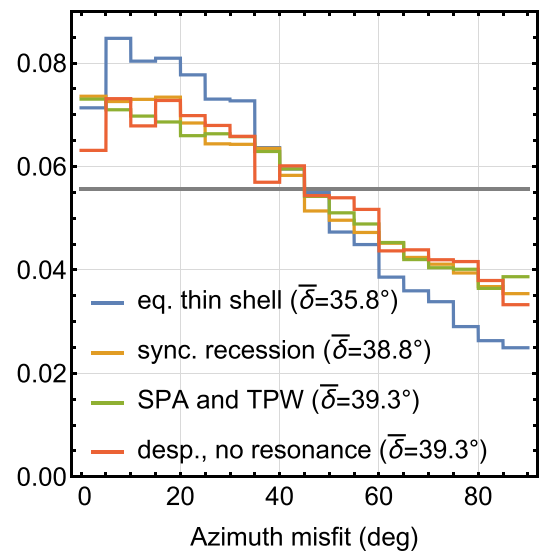

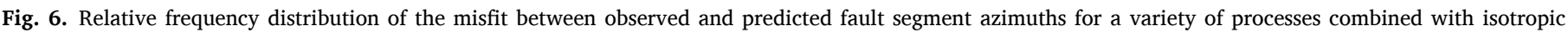

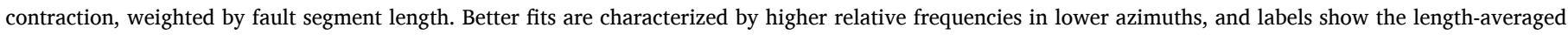

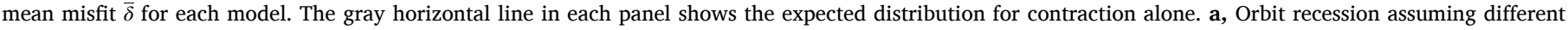

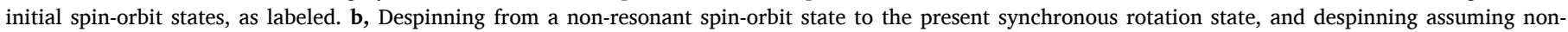

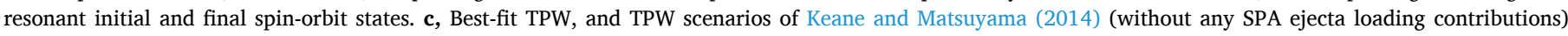

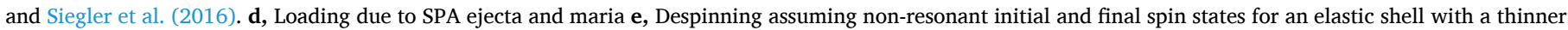

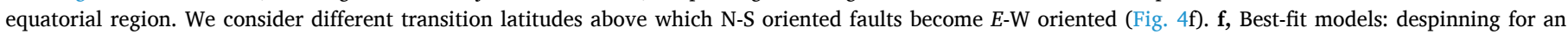

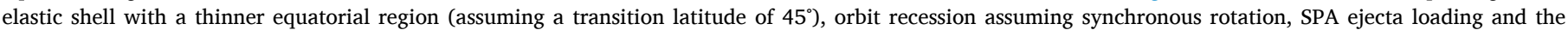
corresponding TPW, and despinning with non-resonant initial and final spin-orbit states.

stresses for recession, despinning, and SPA ejecta loading and the corresponding TPW (Fig. 7).

\section{Conclusions}

The global thrust fault pattern of the Moon has been explained as due to the combination of global contraction with orbit recession and diurnal tidal stresses (Watters et al. 2015a, 2019). In this paper, we illustrate that global contraction combined with other processes can provide an alternative explanation. In particular, global contraction combined with orbit recession with different initial spin-orbit states, despinning from a non-resonant spin-orbit state to another non-resonant one, and SPA ejecta loading with the corresponding TPW result in equally good model fits (Fig. 6).

The model presented in this paper is general enough to consider multiple stress-generating processes, including mass loading with arbitrary geometry, rotational and orbital perturbations, and arbitrary elastic lithosphere thicknesses. One limitation of the model is that it assumes an elastic lithosphere with constant thickness. We consider the tectonic patterns on a non-uniform, thin elastic shell using the approach of Beuthe (2010). Contraction and despinning assuming non-resonant initial and final spin states for an elastic shell with a thinner equatorial region can lead to misfits that are smaller than those discussed above assuming a constant thickness elastic shell (Fig. 6f).

The young age of the population of thrust fault scarps favors recent contraction and recession. However, unrelaxed stresses from older processes combined with recent contractional stresses can also generate young faults. The non-hydrostatic figure of the Moon suggests a fossil figure (Jeffreys 1915; Lambeck and Pullan 1980; Garrick-Bethell et al. 2006; Matsuyama 2013; Keane and Matsuyama 2014; Qin et al. 2018), which requires the preservation of ancient stresses. One of the best-fit solutions discussed above, SPA ejecta loading and the corresponding TPW, includes a self-consistent computation of a reoriented fossil figure contribution ( $\$ 2.1 .1)$, combined with a large $(\gtrsim 1 \mathrm{~km})$ isotropic radius 
a

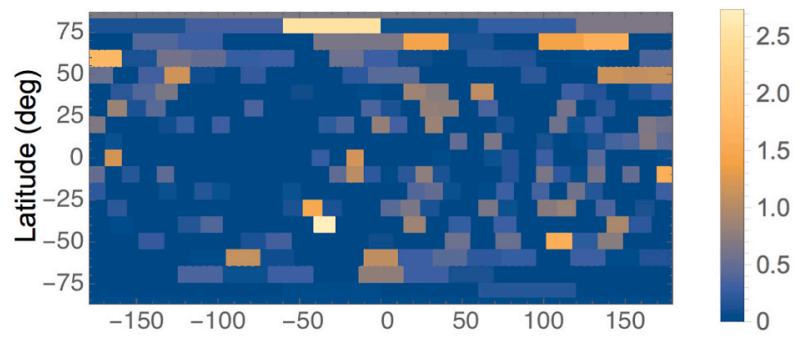

C

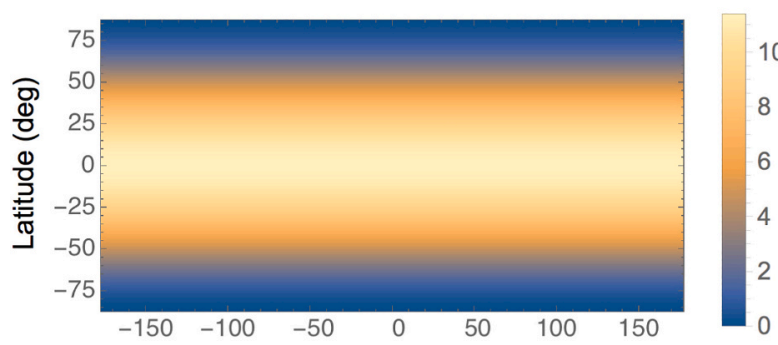

e

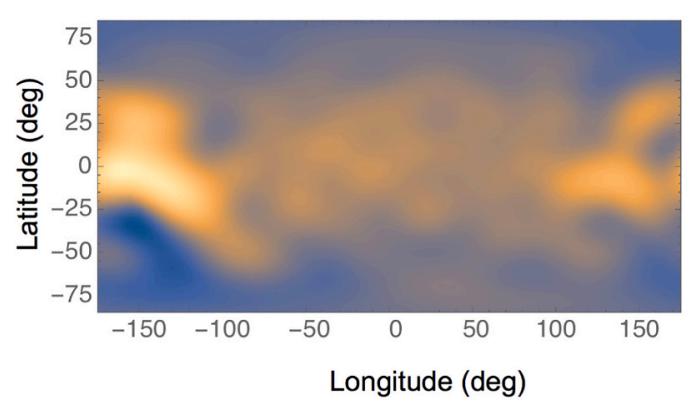

$10^{3} \mathrm{~km}^{-1}$

$\mathrm{MPa}$

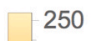

b

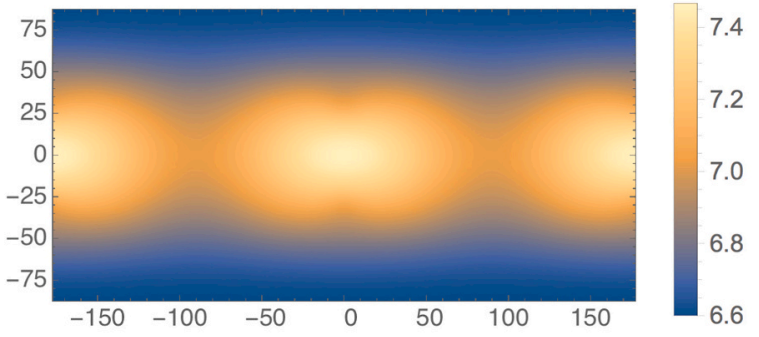

d

Despinning (vaiable shell)

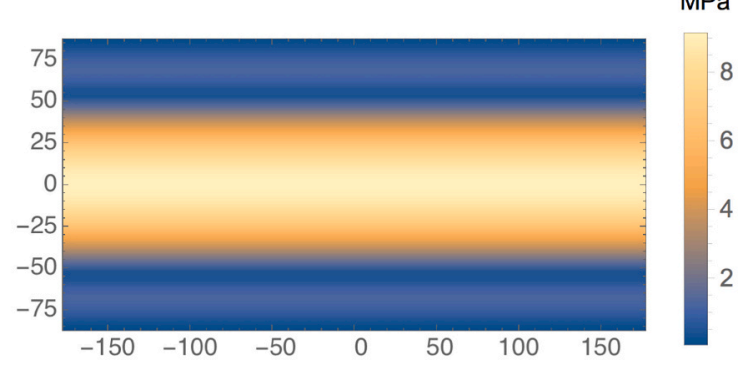

f

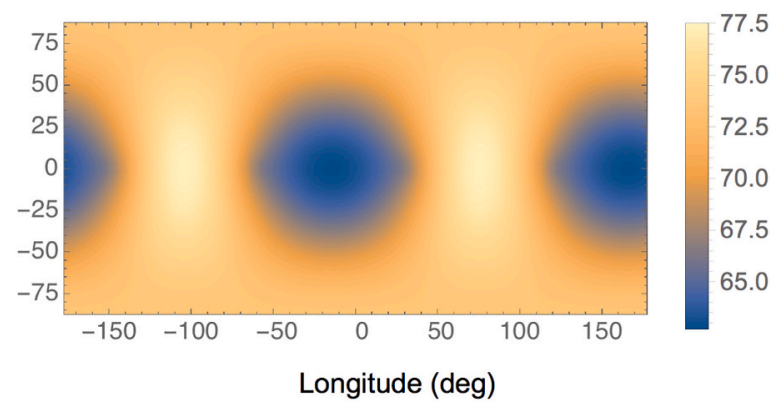

Fig. 7. a, Total fault segment length per unit area computed by sampling the digitized fault segments in 400 equal area regions partitioned using the "igloo" method of Leopardi (2006). b-f, Absolute difference between the maximum and minimum principal stresses (principal stresses difference), which quantifies the deviatoric stress, for a variety of mechanisms combined with isotropic contraction (b, orbit recession assuming synchronous rotation throughout; $\mathbf{c}$, despinning assuming nonresonant initial and final spin states and a uniform shell; $\mathbf{d}$, same as panel c, but for a shell with a thinner equatorial region assuming $\pm 35^{\circ}$ latitudes for the transition between N-S and E-W predicted faults; e, SPA ejecta loading and the corresponding TPW; and f, best-fit TPW). Despinning scenarios assume rotation rate variations alone without orbit recession. For recession and despinning, the magnitude of the principal stresses difference is somewhat arbitrary because the amount of recession and despinning is unconstrained; however, the spatial pattern shown in panels b-d is independent of these parameters. 
contraction to make horizontal principal stresses compressional on the entire surface and generate global thrust faulting. Despinning assuming an elastic shell with a thinner equatorial region provides a smaller misfit to the observed pattern; however, despinning stresses are smaller than fossil figure stresses. The minimum radius contraction of $\sim 1 \mathrm{~km}$ required to overcome large fossil figure stresses and generate global thrust faulting corresponds to the total contraction since the fossil figure is established, the timing of which is uncertain. It is worth noting that this minimum radius contraction is significantly larger than the radius contraction of $\gtrsim 25 \mathrm{~m}$ over the past $\sim 100$ Myr inferred from strain estimates based on the population of fault scarps (Watters et al. 2010, 2015a). Ultimately, distinguishing between the scenarios described above requires time-dependent stress evolution models with the age of the faults as additional constraints, which is challenging given the limited constraints on time-dependent model parameters.

\section{Data Availability}

Data analyzed in this study were a re-analysis of existing data, which are openly available at locations cited in the reference section. Reanalyzed data are available at the University of Arizona's Research Data Repository (https://doi.org/10.25422/azu.data.12935162).

\section{Declaration of Competing Interest}

None.

\section{Acknowledgments}

We thank P. James and K. Crane for their thoughtful comments and suggestions that improved the manuscript. This material is based upon work supported by the National Aeronautics and Space Administration (NASA) under Grant No. 80NSSC17K0724 issued through the NASA Solar System Workings program. M. Beuthe was supported by the Belgian PRODEX program managed by the European Space Agency in collaboration with the Belgian Federal Science Policy Office. T. R. W. was supported by the LRO Project and an ASU LROC contract.

\section{A. Stress tensor}

Assuming an isotropic linear elastic solid (e.g. Jaeger et al. 2009, Eq. (5.16)),

$\tau_{i j}=\lambda \delta_{i j} \varepsilon_{k k}+2 \mu \varepsilon_{i j}$,

where the strain components in spherical coordinates are (Dahlen and Tromp, 1998, Eq. A.139)

$$
\begin{aligned}
\varepsilon_{r r} & =\partial_{r} d_{r} \\
\varepsilon_{\theta \theta} & =\frac{1}{r}\left(\partial_{\theta} d_{\theta}+d_{r}\right) \\
\varepsilon_{\phi \phi} & =\frac{1}{r \sin \theta}\left(\partial_{\phi} d_{\phi}+d_{r} \sin \theta+d_{\theta} \cos \theta\right)=\frac{1}{r}\left(\frac{\partial_{\phi} d_{\phi}}{\sin \theta}+d_{r}+d_{\theta} \cot \theta\right) \\
\varepsilon_{\theta \phi} & =\frac{1}{2 r}\left(\frac{\partial_{\phi} d_{\theta}}{\sin \theta}+\partial_{\theta} d_{\phi}-d_{\phi} \cot \theta\right)
\end{aligned}
$$

\section{A.1. Compressible interior}

For a compressible interior, it is useful to write the other components of the (Lagrangian increment to the Cauchy) stress tensor in terms of $\tau_{r r}$ using the relation

$\partial_{r} d_{r}=\frac{\tau_{r r}}{(\lambda+2 \mu)}-\frac{\lambda}{(\lambda+2 \mu) R}\left(\partial_{\theta} d_{\theta}+2 d_{r}+\frac{\partial_{\phi} d_{\phi}}{\sin \theta}+d_{\theta} \cot \theta\right)$

derived from Eqs. (19) and (20). We obtain Eq. (13) in the main text for the stress tensor by combining Eqs. (19)-(21).

Combining Eqs. (12) and (13),

$$
\begin{gathered}
\tau_{\theta \theta}=-\frac{\lambda}{(\lambda+2 \mu)} \tau_{r r}+\frac{2 \mu}{(\lambda+2 \mu)} \sum_{\ell=0}^{\infty} \sum_{m=-\ell}^{\ell}\left[h_{\ell}(3 \lambda+2 \mu)+l_{\ell}\left(\frac{\lambda}{\sin ^{2} \theta} \partial_{\phi}^{2}+\lambda \cot \theta \partial_{\theta}+2(\lambda+\mu) \partial_{\theta}^{2}\right)\right] \Phi_{\ell m} Y_{\ell m} \\
\tau_{\phi \phi}=-\frac{\lambda}{(\lambda+2 \mu)} \tau_{r r}+\frac{2 \mu}{(\lambda+2 \mu)} \sum_{\ell=0}^{\infty} \sum_{m=-\ell}^{\ell}\left[h_{\ell}(3 \lambda+2 \mu)+l_{\ell}\left(\frac{2(\lambda+\mu)}{\sin ^{2} \theta} \partial_{\phi}^{2}+2(\lambda+\mu) \cot \theta \partial_{\theta}+\lambda \partial_{\theta}^{2}\right)\right] \Phi_{\ell m} Y_{\ell m} \\
\tau_{\theta \phi}=\frac{2 \mu}{\sin \theta} \sum_{\ell=0}^{\infty} \sum_{m=-\ell}^{\ell} l_{\ell}\left[\partial_{\theta} \partial_{\phi}-\cot \theta \partial_{\phi}\right] \Phi_{\ell m} Y_{\ell m} .
\end{gathered}
$$

These equations are equivalent to Eqs. (B.11)-(B.13) in Wahr et al. (2009) for rotational or tidal stresses at the surface with $\tau_{r r}=0$.

\section{A.2. Incompressible interior}

The predicted tectonic patterns are not sensitive to the assumption of an incompressible interior. Therefore, mass loading, recession, despinning, 
and TPW stresses can be computed assuming an incompressible interior, which involves incompressible Love numbers that are easier to compute. In this case, we combine the incompressible condition $\nabla \cdot \mathbf{d}=0$,

$\partial_{r} d_{r}=-\frac{1}{r}\left(2 d_{r}+\partial_{\theta} d_{\theta}+\cot \theta d_{\theta}+\frac{1}{\sin \theta} \partial_{\phi} d_{\phi}\right)$

with

$\tau_{r r}=\lambda \varepsilon_{k k}+2 \mu \varepsilon_{r r}=\lambda \varepsilon_{k k}+2 \mu \partial_{r} d_{r}$

to obtain

$\lambda \varepsilon_{k k}=\tau_{r r}+\frac{2 \mu}{r}\left(2 d_{r}+\partial_{\theta} d_{\theta}+\cot \theta d_{\theta}+\frac{1}{\sin \theta} \partial_{\phi} d_{\phi}\right)$

In the last equation, $\lambda \rightarrow \infty, \varepsilon_{k k}=\nabla \cdot \mathbf{d}=0$, and the product $\lambda \varepsilon_{k k}$ remains finite. Using Eqs. (19), (20), and (25), the non-zero stress tensor components can be written as

$$
\begin{aligned}
\tau_{\theta \theta} & =\tau_{r r}+\frac{6 \mu}{r} d_{r}+\frac{2 \mu}{r}\left[2 \partial_{\theta} d_{\theta}+\cot \theta d_{\theta}+\frac{1}{\sin \theta} \partial_{\phi} d_{\phi}\right] \\
\tau_{\phi \phi} & =\tau_{r r}+\frac{6 \mu}{r} d_{r}+\frac{2 \mu}{r}\left[\partial_{\theta} d_{\theta}+2 d_{\theta} \cot \theta+2 \frac{\partial_{\phi} d_{\phi}}{\sin \theta}\right] \\
\tau_{\theta \phi} & =\frac{\mu}{R}\left(\partial_{\theta} d_{\phi}+\frac{\partial_{\phi} d_{\theta}}{\sin \theta}-d_{\phi} \cot \theta\right)
\end{aligned}
$$

Combining Eqs. (12) and (26),

$$
\begin{aligned}
& \tau_{\theta \theta}=\tau_{r r}+2 \mu \sum_{\ell=0}^{\infty} \sum_{m=-\ell}^{\ell}\left[3 h_{\ell}+l_{\ell}\left(2 \partial_{\theta}^{2}+\cot \theta \partial_{\theta}+\frac{1}{\sin ^{2} \theta} \partial_{\phi}^{2}\right)\right] \Phi_{\ell m} Y_{\ell m} \\
& \tau_{\phi \phi}=\tau_{r r}+2 \mu \sum_{\ell=0}^{\infty} \sum_{m=-\ell}^{\ell}\left[3 h_{\ell}+l_{\ell}\left(\partial_{\theta}^{2}+2 \cot \theta \partial_{\theta}+\frac{2}{\sin ^{2} \theta} \partial_{\phi}^{2}\right)\right] \Phi_{\ell m} Y_{\ell m} . \\
& \tau_{\theta \phi}=\frac{\mu}{\sin \theta} \sum_{\ell=0}^{\infty} \sum_{m=-\ell}^{\ell} l_{\ell}\left(2 \partial_{\theta} \partial_{\phi}-2 \cot \theta \partial_{\phi}\right) \Phi_{\ell m} Y_{\ell m} .
\end{aligned}
$$

The Love numbers in Eqs. (22) and (27) must be used self-consistently to obtain the correct stress tensor. That is, Love numbers computed assuming a compressible interior must be used in Eq. (22), whereas Love numbers computed assuming an incompressible interior must be used in Eq. (27).

\section{B. Spherical harmonics identities}

The spherical harmonic identities below are useful for evaluating the derivatives in Eqs. (22) and (27).

$\frac{1}{\sin \theta} \partial_{\theta}\left(\sin \theta \partial_{\theta} P_{\ell m}\right)=\partial_{\theta}^{2} P_{\ell m}+\cot \theta \partial_{\theta} P_{\ell m}=\left[-\ell(\ell+1)+\frac{m^{2}}{\sin ^{2} \theta}\right] P_{\ell m}$

(Arfken and Weber 1995, Eq. 12.72, p. 722), and

$\partial_{\theta} P_{\ell m}=-\sin \theta \partial_{x} P_{\ell m}=\frac{1}{2}(\ell+m)(\ell+1-m) P_{\ell, m-1}-\frac{1}{2} P_{\ell, m+1}=\frac{1}{2}\left(A_{\ell m} P_{\ell, m-1}-P_{\ell, m+1}\right)$,

(Arfken and Weber 1995, Eq. 12.72, p. 722), where $x \equiv \cos \theta$ and we define

$A_{\ell m} \equiv(\ell+m)(\ell-m+1)$

The second derivative can be written as

$\partial_{\theta}^{2} P_{\ell m}=\frac{1}{4}\left[B_{\ell m} P_{\ell m}+C_{\ell m} P_{\ell, m-2}+P_{\ell, m+2}\right]$

where we define

$B_{\ell m} \equiv-A_{\ell m}-A_{\ell m+1}$

$C_{\ell m} \equiv A_{\ell m} A_{\ell, m-1}$. 
a

Tidal Love numbers

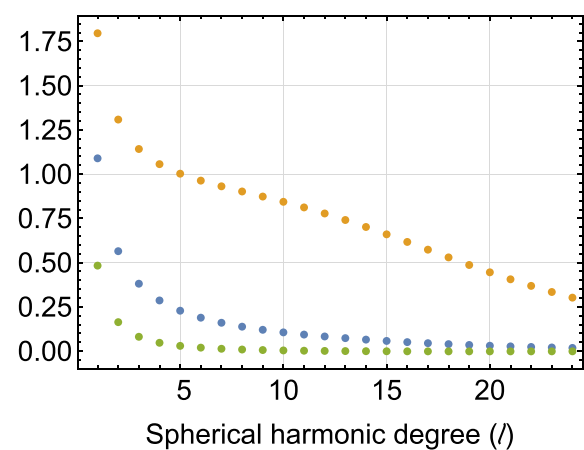

C

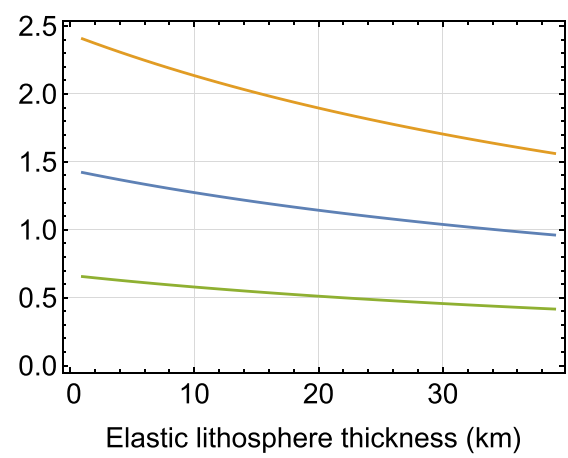

e

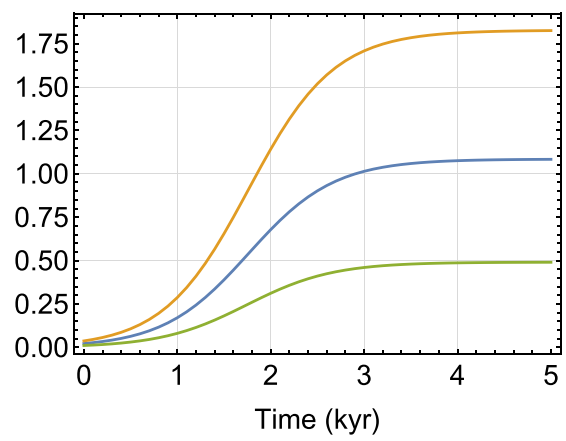

b

Load Love numbers
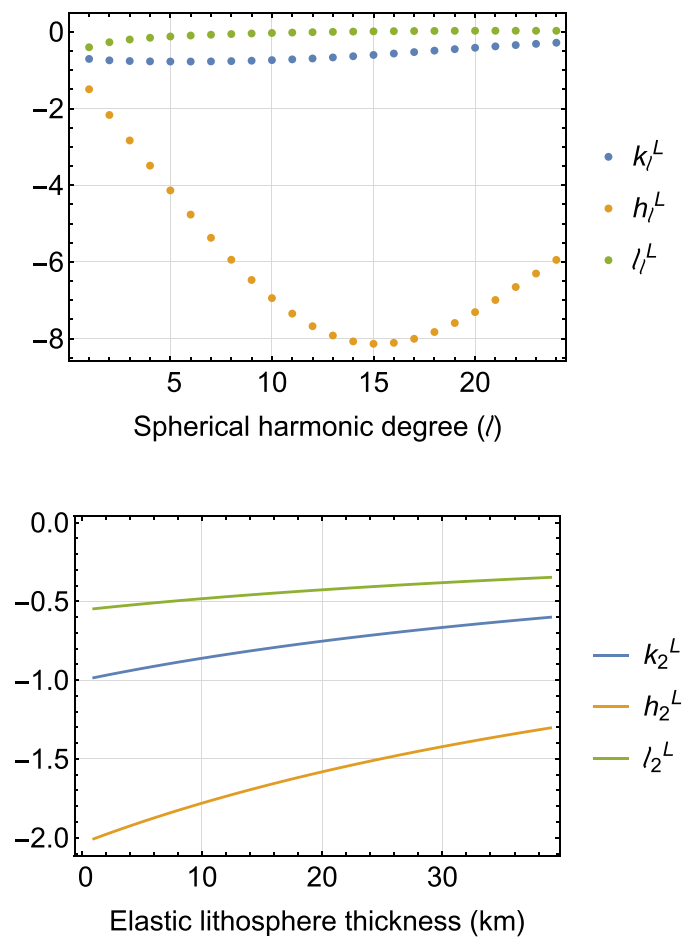

f d

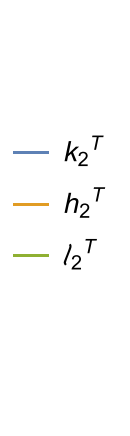

$k_{l}^{T}$
$-h_{l}^{T}$
$-l_{l}^{T}$

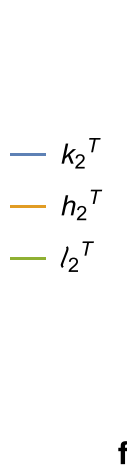

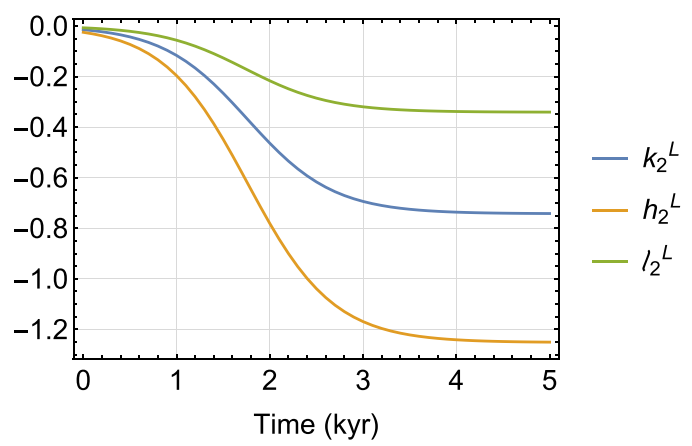

Fig. 8. Top: (a) tidal and (b) load Love numbers as a function of spherical harmonic degree. Middle: Degree-2 (c) tidal and (d) load Love numbers as a function of elastic lithosphere thickness. Bottom: Time-dependent degree-2 (e) tidal and (f) load Love numbers as a function time assuming a step-function forcing at time $t=0$. We assume the Moon parameters adopted in this paper (Table 1). Additionally, computing the time-dependent Love numbers requires specifying the rigidity and viscosity of all layers, and we assume a core rigidity and viscosity of $40 \mathrm{GPa}$ and $10^{21} \mathrm{~Pa} \mathrm{~s}$, a mantle rigidity and viscosity of $70 \mathrm{GPa}$ and $10^{21} \mathrm{~Pa}$ s, and a lithosphere rigidity and viscosity of $40 \mathrm{GPa}$ and $10^{26} \mathrm{~Pa}$.

\section{Love numbers}

Tidal and load Love numbers can be computed by solving mass and momentum conservation, and Poisson's equation at each interior layer, which is commonly done using the propagator matrix method (e.g. Sabadini et al. 2016). Across any incompressible layer (we will assume all layers are incompressible except the elastic lithosphere), a full set of linearly independent analytical solutions can be found, the boundary values of which we use to build the propagator matrix. Across the compressible elastic lithosphere, no analytical solution can be found (unless the density were uniform throughout the body, making $g / r$ a constant (Gilbert and Backus 1968), and we obtain the propagator matrix by numerical integration over a full set of linearly independent "initial" (i.e. bottom) values.

The computation of the degree-1 Love numbers requires a special treatment (Farrell 1972; Okubo and Endo 1986; Greff-Lefftz and Legros 1997). The non-existence of a static solution under a degree- 1 tide-generating field (a spatially uniform field) forbids $\ell=1$ tidal Love numbers. An additional boundary condition is required for the $\ell=1$ load Love numbers, and we choose to solve the loading problem in a set of axes originating at the center of mass, where the degree-1 loading-induced incremental potential cancels the degree-1 loading potential, so that the total degree- 1 potential remains zero. That is, $k_{1}^{L}=-1$, which provides the missing independent boundary condition for degree-1 loading (Greff-Lefftz and Legros 1997). We use the classical propagator matrix method (e.g. Sabadini et al. 2016) with this additional boundary condition to compute the $\ell=1$ load Love numbers.

Fig. 8 shows the tidal and load Love numbers for the Moon parameters adopted in this paper (Table 1), and the time-dependent Love numbers assuming a step-function forcing at time $t=0$. In practice, this is done by computing static Love numbers but with a time-dependent (exponentially decaying) rigidity. The time-dependent Love numbers converge to the long-term Love numbers, as expected, with a convergence time of a few kyr. 
Therefore, the use of long-term Love numbers is justified when considering stresses and tectonic patterns over timescales longer than a few kyr. The long-term Love numbers are computed by assuming that all interior layers except the elastic lithosphere behave fluidly, that is, by assuming zero rigidity for all layers except the elastic lithosphere (Table 1).

\section{Comparison with previous work}

We validate our model by comparing our results with those of previous studies on despinning (Melosh 1977), TPW (Melosh 1980), and surface mass loading (Janes and Melosh 1990). These studies often use a thin shell approximation to simplify the solutions (Vening-Meinesz 1947; Melosh 1980; Matsuyama and Nimmo 2008). Our method is general enough to consider these mechanisms with the same formalism. Additionally, our method is not limited by assumptions about the interior structure, including the thickness of the elastic lithosphere.

The thick shell solutions presented here explicitly reduce to thin shell approximation solutions (e.g. Matsuyama and Nimmo 2008) by using the $\ell_{n}$ $-h_{n}$ Love numbers relation for an elastic membrane (Beuthe 2015, Eqs. (65) and (77)).

\section{E. Deviatoric stresses due to diurnal forcing}

Previous studies considering diurnal stresses compute the expected tectonic pattern using the stresses at pericenter (Watters et al. 2015a, 2019). However, deviatoric stresses are maximum at pericenter only for a small fraction of the surface, as illustrated in Fig. 9. Different points on the surface experience maximum deviatoric stresses at different times, complicating the calculation of the predicted tectonic pattern. It is possible to calculate the tectonic pattern by defining a failure stress and considering the principal stresses at the time when the deviatoric stress becomes equal to the failure stress (or a similar failure criteria) at different surface locations.

a $0^{\circ} \mathrm{E}$

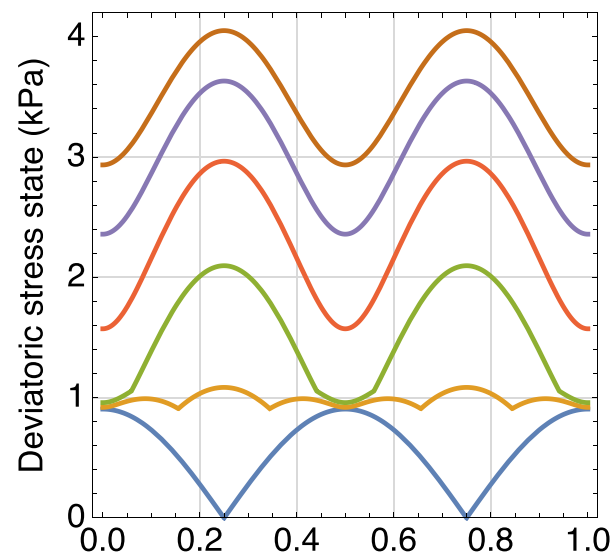

C

$60^{\circ} \mathrm{E}$

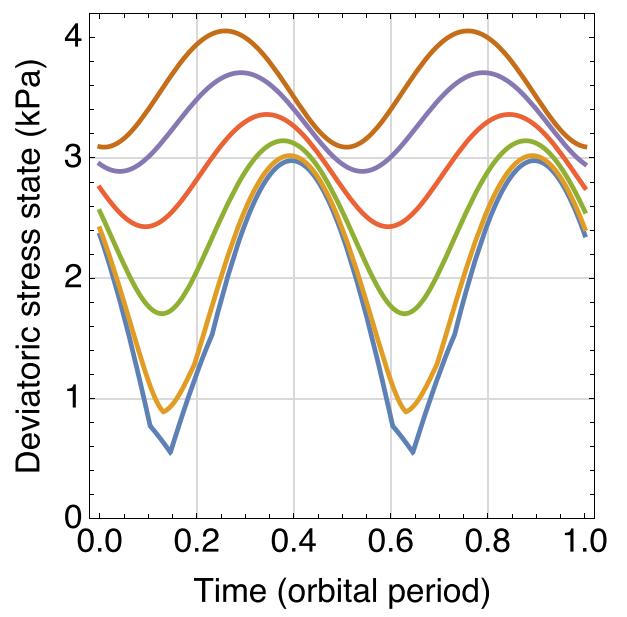

$30^{\circ} \mathrm{E}$

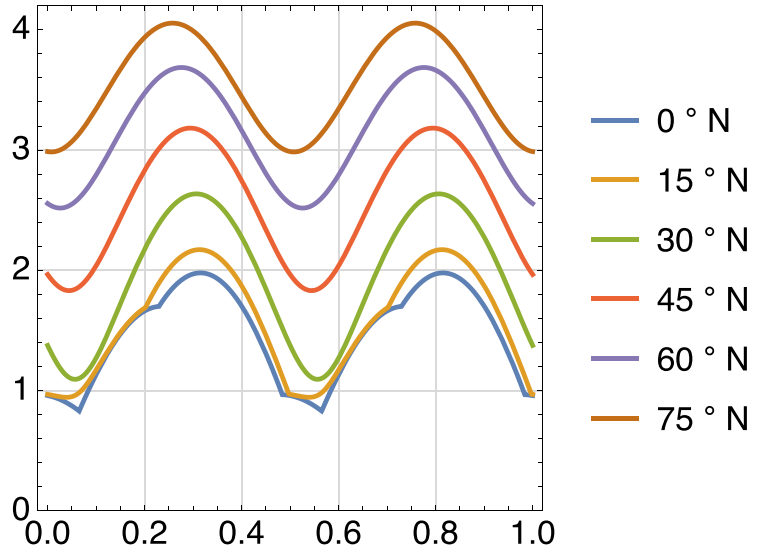

$90^{\circ} \mathrm{E}$

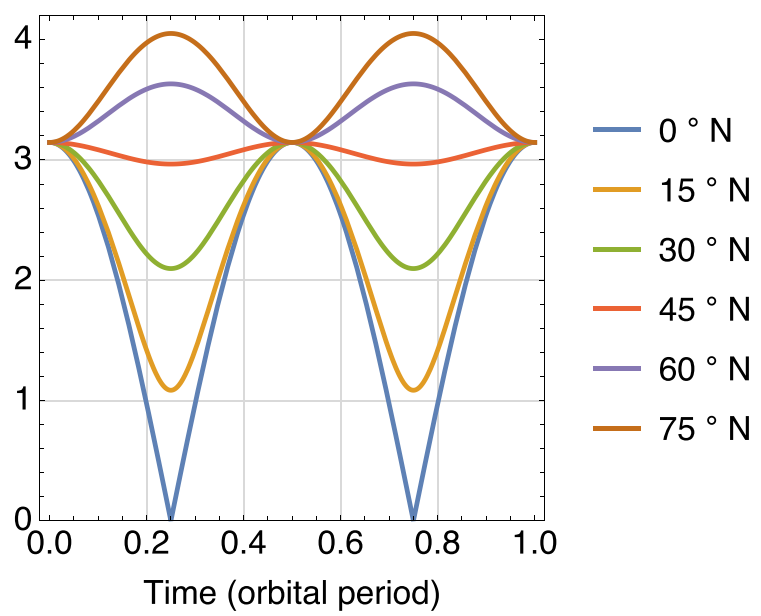

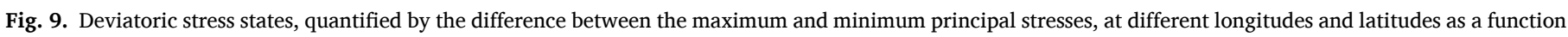

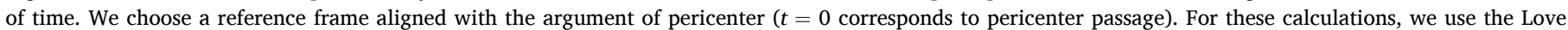
numbers at the tidal forcing period.

The relative magnitude of diurnal and recession stresses can be estimated using the corresponding radial deformation $d_{r}$. For recession, 
$d_{r} \sim h_{2}^{T} \frac{M_{T}}{M} R^{3}\left(\frac{1}{a_{i}^{3}}-\frac{1}{a^{3}}\right)$,

where $h_{2}^{T}$ is the long-term displacement Love number and $a_{i}$ is the initial semimajor axis. For diurnal tides,

$d_{r} \sim e h_{2}^{T}(t=T) \frac{M_{T}}{M} \frac{R^{3}}{a^{3}}$,

where $e$ is the orbital eccentricity and $h_{2}^{T}(t=T)$ is the displacement Love number at the tidal forcing period $T$. This time-dependent Love number must be computed assuming a periodic forcing and is therefore different from the one shown in Fig. 8e for a step-function forcing. This order of magnitude estimate yields recession stresses that are 4 orders of magnitude larger than diurnal stresses for an initial semimajor axis $a_{i} \sim 18$ Earth radii that is consistent with the presence of a fossil figure today (Matsuyama 2013; Keane and Matsuyama 2014; Qin et al. 2018), exceeding the typical rock failure stresses $\sim 1 \mathrm{MPa}$. Even if we limit orbit recession to the last $\sim 100 \mathrm{Myr}$ and assume the present recession rate $\sim 4 \mathrm{~cm} \mathrm{yr}^{-1}$, recession stresses remain $\sim 30$ times larger than diurnal stresses. The small orbital eccentricity and the larger deformation over longer time scales, quantified by the difference between the displacement Love number at the tidal forcing period and the recession period, $h_{2}^{T} / h_{2}^{T}(t=T) \sim 50$, contributes significantly to the difference in the deviatoric stresses. Deviatoric stresses for the SPA ejecta loading and the corresponding TPW are also larger than the diurnal deviatoric stresses by 4 orders of magnitude, also exceeding the typical rock failure stresses $\sim 1 \mathrm{MPa}$ (Fig. 7 ).

\section{References}

Anderson, E.M., 1951. The Dynamics of Faulting. Oliver \& Boyd, Edinburg.

Andrews-Hanna, J.C., Besserer, J., Head, J.W.I., Howett, C.J.A., Kiefer, W.S., Lucey, P.J., McGovern, P.J., Melosh, H.J., Neumann, G.A., Phillips, R.J., Schenk, P.M., Smith, D. E., Solomon, S.C., Zuber, M.T., 2014. Structure and evolution of the lunar Procellarum region as revealed by GRAIL gravity data. Nature 514, 68-71.

Arfken, G., Weber, H., 1995. Mathematical Methods for Physicists. Academic Press, Fourth ed.

Banerdt, W.B., Phillips, R.J., Sleep, N.H., Saunders, R.S., 1982. Thick shell tectonics on one-plate planets: applications to Mars. J. Geophys. Res. 87, 9723-9733.

Banks, M.E., Watters, T.R., Robinson, M.S., Tornabene, L.L., Tran, T., Ojha, L., Williams, N.R., 2012. Morphometric analysis of small-scale lobate scarps on the Moon using data from the Lunar Reconnaissance Orbiter. J. Geophys. Res. 117. E0OH11-n/a.

Beuthe, M., 2010. East-west faults due to planetary contraction. Icarus 209, 795-817.

Beuthe, M., 2013. Spatial patterns of tidal heating. Icarus 223, 308-329.

Beuthe, M., 2015. Tidal Love numbers of membrane worlds: Europa, Titan, and Co. Icarus 258, 239-266.

Beuthe, M., 2018. Enceladus's crust as a non-uniform thin shell: I tidal deformations. Icarus 302, 145-174.

Binder, A.B., 1982. Post-Imbrian global lunar tectonism - evidence for an initially totally molten moon. Moon and the Planets 26, 117-133.

van der Bogert, C.H., Clark, J.D., Hiesinger, H., Banks, M.E., Watters, T.R., Robinson, M. S., 2018. How old are lunar lobate scarps? 1. Seismic resetting of crater sizefrequency distributions. Icarus 306, 225-242.

Bouley, S., Keane, J.T., Baratoux, D., Langlais, B., Matsuyama, I., Costard, F., Hewins, R., Payré, V., Sautter, V., Séjourné, A., Vanderhaeghe, O., Zanda, B., 2020. A thick crustal block revealed by reconstructions of early Mars highlands. Nat. Geosci. 13, 105-109.

Cheng, C.H., Toksoz, M.N., 1978. Tidal stresses in the moon. J. Geophys. Res. 83, 845-853.

Clark, J.D., Hurtado, J.M., Hiesinger, H., van der Bogert, C.H., Bernhardt, H., 2017. Investigation of newly discovered lobate scarps: implications for the tectonic and thermal evolution of the Moon. Icarus 298, 78-88.

Dahlen, F.A., Tromp, J., 1998. Theoretical Global Seismology. Princeton University Press.

Dickey, J.O., Bender, P.L., Faller, J.E., Newhall, X.X., Ricklefs, R.L., Ries, J.G., Shelus, P.J., Veillet, C., Whipple, A.L., Wiant, J.R., Williams, J.G., Yoder, C.F., 1994. Lunar laser ranging: a continuing legacy of the Apollo program. Science 265, 482-490.

Farrell, W.E., 1972. Deformation of the earth by surface loads. Rev. Geophys. Space Phys. 10, 761-797.

Fisher, N., 1995. Statistical Analysis of Circular Data. Statistical Analysis of Circular Data. Cambridge University Press. URL. https://books.google.com/books?id=wGP j3EoFdJwC.

Garrick-Bethell, I., Wisdom, J., Zuber, M.T., 2006. Evidence for a past high-eccentricity lunar orbit. Science 313, 652-655.

Garrick-Bethell, I., Nimmo, F., Wieczorek, M.A., 2010. Structure and formation of the lunar Farside highlands. Science 330, 949-951.

Garrick-Bethell, I., Perera, V., Nimmo, F., Zuber, M.T., 2014. The tidal-rotational shape of the moon and evidence for polar wander. Nature 1-4.

Gilbert, F., Backus, G., 1968. Elastic-gravitational vibrations of a radially stratified sphere. In: Herrmann, G. (Ed.), Dynamics of Stratified Solids. American Society of Mechanical Engineers, New York, pp. 82-95.

Goldreich, P., 1966. History of the lunar orbit. Rev. Geophys. Space Phys. 4, 411-439.

Gong, S., Wieczorek, M.A., Nimmo, F., Kiefer, W.S., Head, J.W., Huang, C., Smith, D.E. Zuber, M.T., 2016. Thicknesses of mare basalts on the moon from gravity and topography. J. Geophys. Res. 121, 854-870.

Greff-Lefftz, M., Legros, H., 1997. Some remarks about the degree-one deformation of the earth. Geophys. J. 131, 699-723.
Harada, Y., Kurita, K., 2006. The dependence of surface tidal stress on the internal structure of Europa: the possibility of cracking of the icy shell. Planet. Space Sci. 54, $170-180$.

Jaeger, J., Cook, N., Zimmerman, R., 2009. Fundamentals of Rock Mechanics. Wiley. URL. https://books.google.com/books?id=PbMqM8GH9-IC.

Janes, D.M., Melosh, H.J., 1990. Tectonics of planetary loading: a general model and results. J. Geophys. Res. 95, 21345-21355.

Jeffreys, H., 1915. Certain hypothesis as to the internal structure of the earth and moon. Mem. Royal Astro. Soc. 60, 187-217.

Jolliff, B.L., Gillis, J.J., Haskin, L.A., Korotev, R.L., Wieczorek, M.A., 2000. Major lunar crustal terranes: surface expressions and crust-mantle origins. J. Geophys. Res. 105, 4197-4216.

Keane, J.T., Matsuyama, I., 2014. Evidence for lunar true polar wander, and a past loweccentricity, synchronous lunar orbit. Geophys. Res. Lett. 41, 6610-6619.

Konopliv, A.S., Park, R.S., Yuan, D.N., Asmar, S.W., Watkins, M.M., Williams, J.G., Fahnestock, E., Kruizinga, G., Paik, M., Strekalov, D., Harvey, N., Smith, D.E., Zuber, M.T., 2013. The JPL lunar gravity field to spherical harmonic degree 660 from the GRAIL Primary Mission. J. Geophys. Res. 118, 1415-1434.

Lambeck, K., Pullan, S., 1980. The lunar fossil bulge hypothesis revisited. Phys. Earth Planet. Inter. 22, 29-35.

Leopardi, P., 2006. A partition of the unit sphere into regions of equal area and small diameter. Electron. Trans. Numer. Anal. 25, 309-327.

Matsuyama, I., 2013. Fossil figure contribution to the lunar figure. Icarus 222, 411-414.

Matsuyama, I., Nimmo, F., 2008. Tectonic patterns on reoriented and despun planetary bodies. Icarus 195, 459-473.

Matsuyama, I., Nimmo, F., 2009. Gravity and tectonic patterns of Mercury: effect of tidal deformation, spin-orbit resonance, nonzero eccentricity, despinning, and reorientation. J. Geophys. Res. 114, E01010.

Matsuyama, I., Nimmo, F., Mitrovica, J.X., 2014. Planetary reorientation. Annu. Rev, Earth Planet. Sci. 42, 605-634.

Matsuyama, I., Nimmo, F., Keane, J.T., Chan, N.H., Taylor, G.J., Wieczorek, M.A., Kiefer, W.S., Williams, J.G., 2016. GRAIL, LLR, and LOLA constraints on the interior structure of the Moon. Geophys. Res. Lett. 43, 8365-8375.

Melosh, H.J., 1975. Mascons and the moon's orientation. Earth Planet. Sci. Lett. 25, 322-326.

Melosh, H.J., 1977. Global tectonics of a despun planet. Icarus 31, 221-243.

Melosh, H.J., 1980. Tectonic patterns on a reoriented planet - Mars. Icarus 44, 745-751.

Melosh, H.J., Kendall, J., Horgan, B., Johnson, B.C., Bowling, T., Lucey, P.G., Taylor, G. J., 2017. South Pole-Aitken basin ejecta reveal the Moon's upper mantle. Geology 45, 1063-1066.

Meyer, J., Elkins-Tanton, L., Wisdom, J., 2010. Coupled thermal-orbital evolution of the early Moon. Icarus 208, 1-10.

Murray, C., Dermott, S., 1999. Solar system dynamics. Cambridge University Press.

Nelson, D.M., Koeber, S.D., Daud, K., Robinson, M.S., Watters, T.R., Banks, M.E., Williams, N.R., 2014. Mapping Lunar Maria Extents and Lobate Scarps Using Lroc Image Products. 45th Lunar and Planetary Science Conference, 45, p. 2861.

Neumann, G.A., Zuber, M.T., Wieczorek, M.A., Head, J.W., Baker, D.M.H., Solomon, S.C., Smith, D.E., Lemoine, F.G., Mazarico, E.M., Sabaka, T.J., Goossens, S.J., Melosh, H. J., Phillips, R.J., Asmar, S.W., Konopliv, A.S., Williams, J.G., Sori, M.M., Soderblom, J.M., Miljkovic, K., Andrews-Hanna, J.C., Nimmo, F., Kiefer, W.S., 2015. Lunar impact basins revealed by Gravity Recovery and Interior Laboratory measurements. Sci. Adv. 1, e1500852.

Okubo, S., Endo, T., 1986. Static spheroidal deformation of degree 1 - consistency relation, stress solution and partials. Geophys. J. 86, 91-102.

Peale, S.J., Cassen, P., 1978. Contribution of tidal dissipation to lunar thermal history. Icarus 36, 245-269.

Qin, C., Zhong, S., Phillips, R., 2018. Formation of the lunar fossil bulges and its implication for the early earth and moon. Geophys. Res. Lett. 45, 1286-1296.

Sabadini, R., Vermeersen, B., Cambiotti, G., 2016. Global Dynamics of the Earth: Applications of Viscoelastic Relaxation Theory to Solid-Earth and Planetary Geophysics. Springer Netherlands. URL. https://books.google.com/books?id=33x BDAAAQBAJ. 
Schultz, P.H., 1976. Moon Morphology: Interpretations Based on Lunar Orbiter Photography. University of Texas Press, Austin.

Siegler, M.A., Miller, R.S., Keane, J.T., Laneuville, M., Paige, D.A., Matsuyama, I., Lawrence, D.J., Crotts, A., Poston, M.J., 2016. Lunar true polar wander inferred from polar hydrogen. Nature 531, 480-484.

Solomon, S.C., 1977. The relationship between crustal tectonics and internal evolution in the moon and Mercury. Phys. Earth Planet. Inter. 15, 135-145.

Solomon, S.C., 1986. On the early thermal state of the moon. In: Hartmann, W.K., Phillips, R.J., Taylor, G.J. (Eds.), Origin of the Moon, pp. 311-329.

Solomon, S.C., Chaiken, J., 1976. Thermal expansion and thermal stress in the moon and terrestrial planets - clues to early thermal history. Proc. Lunar Sci. Conf. 7 , 3229-3243.

Turcotte, D.L., Willemann, R.J., Haxby, W.F., Norberry, J., 1981. Role of membrane stresses in the support of planetary topography. J. Geophys. Res. 86, 3951.

Vasavada, A.R., Paige, D.A., Wood, S.E., 1999. Near-surface temperatures on Mercury and the Moon and the stability of polar ice deposits. Icarus 141, 179-193.

Vening-Meinesz, F.A., 1947. Shear Patterns of the Earth's Crust, 28, pp. 1-61.

Wahr, J., Selvans, Z.A., Mullen, M.E., Barr, A.C., Collins, G.C., Selvans, M.M., Pappalardo, R.T., 2009. Modeling stresses on satellites due to nonsynchronous rotation and orbital eccentricity using gravitational potential theory. Icarus 200, 188-206.

Watters, T.R., Robinson, M.S., Beyer, R.A., Banks, M.E., Bell, J.F., Pritchard, M.E., Hiesinger, H., van der Bogert, C.H., Thomas, P.C., Turtle, E.P., Williams, N.R., 2010. Evidence of recent thrust faulting on the moon revealed by the lunar reconnaissance orbiter camera. Science 329, 936-940.
Watters, T.R., Robinson, M.S., Collins, G.C., Banks, M.E., Daud, K., Williams, N.R., Selvans, M.M., 2015a. Global thrust faulting on the Moon and the influence of tidal stresses. Geology 43, 851-854.

Watters, T.R., Selvans, M.M., Banks, M.E., Hauck, S.A., Becker, K.J., Robinson, M.S., 2015b. Distribution of large-scale contractional tectonic landforms on Mercury: implications for the origin of global stresses. Geophys. Res. Lett. 42, 3755-3763.

Watters, T.R., Weber, R.C., Collins, G.C., Howley, I.J., Schmerr, N.C., Johnson, C.L., 2019. Shallow seismic activity and young thrust faults on the Moon. Nat. Geosci. 329, 936-1017.

Wieczorek, M.A., 2015. The gravity and topography of the terrestrial planets. In: Treatise on Geophysics. Elsevier, pp. 165-206.

Wieczorek, M.A., Weiss, B.P., Stewart, S.T., 2012. An impactor origin for lunar magnetic anomalies. Science 335, 1212-1215.

Wieczorek, M.A., Neumann, G.A., Nimmo, F., Kiefer, W.S., Taylor, G.J., Melosh, H.J., Phillips, R.J., Solomon, S.C., Andrews-Hanna, J.C., Asmar, S.W., Konopliv, A.S., Lemoine, F.G., Smith, D.E., Watkins, M.M., Williams, J.G., Zuber, M.T., 2013. The crust of the moon as seen by GRAIL. Science 339, 671-675.

Willemann, R.J., Turcotte, D.L., 1982. The role of lithospheric stress in the support of the Tharsis rise. J. Geophys. Res. 87, 9793-9801.

Williams, G.E., 2000. Geological constraints on the Precambrian history of Earth's rotation and the Moon's orbit. Rev. Geophys. Space Phys. 38, 37-60.

Williams, J.P., Ruiz, J., Rosenburg, M.A., Aharonson, O., Phillips, R.J., 2011. Insolation driven variations of Mercury's lithospheric strength. J. Geophys. Res. 116, E01008.

Zuber, M.T., Smith, D.E., Lemoine, F.G., Neumann, G.A., 1994. The shape and internal structure of the moon from the Clementine Mission. Science 266, 1839. 